\title{
Doppler Imaging of stellar magnetic fields
}

\section{Techniques}

\author{
N. Piskunov and O. Kochukhov \\ Uppsala Astronomical Observatory, Box 515, 75120 Uppsala, Sweden \\ e-mail: oleg@astro.uu.se
}

Received 24 July 2001 / Accepted 22 October 2001

\begin{abstract}
We describe our latest Magnetic Doppler Imaging (MDI) code capable of simultaneously reconstructing the magnetic field vector and chemical composition distribution over the stellar surface. Input data consists of polarization measurements in the line profiles and the reconstruction is performed by solving the regularized inverse problem. The code incorporates the latest developments in the radiative transfer, the optimization and parallel computing techniques. We have also developed a new regularization method that allows application of our MDI code to incomplete data sets (e.g. only intensity and circular polarization) by restricting possible configurations of the field. Numerical experiments to be presented in a forthcoming paper demonstrate what can be achieved with MDI and allow studying of systematic errors that can cause serious distortions of the maps.
\end{abstract}

Key words. stars: magnetic fields - stars: chemically peculiar - radiative transfer - Doppler Imaging

\section{Introduction}

The study of stellar magnetic fields plays a very important role in our understanding of star formation and evolution linking the photosphere to stellar interiors and to the circumstellar medium. The reliable determination of field geometries for a statistically significant number of objects will serve as a crucial test for existing and future stellar models. In particular, the accurate knowledge of the stellar fields is very important for constructing realistic models of star formation and evolution, field generation, physics of the circumstellar medium etc.

The main observational technique for detecting magnetic fields - measurements of polarization of radiation - is quickly maturing. Both broad-band linear polarimetry and spectropolarimetry have been perfected to the level where a polarization of $10^{-3}$ can be reliably detected (Semel et al. 1993) and the new generation of solar polarimeters have achieved an accuracy of $10^{-5}$ (Stenflo et al. 2001). Modelling field geometry even for larger polarization levels is a challenge since many different field configurations may result in identical polarization signatures. One promising technique is Doppler Imaging (DI). The idea of DI originally suggested by Deutsch (1958) has been developed into a powerful tool for mapping abundance distributions on chemically peculiar stars (e.g. Goncharsky et al. 1982). The method allows extraction of the spatial

Send offprint requests to: N. Piskunov,

e-mail: piskunov@astro.uu.se information from rotational modulation of spectral line profiles. The name Doppler Imaging was coined by Steven Vogt and collaborators who were the first to apply it to the mapping of active regions in late-type stars (Vogt et al. 1987). DI has been successfully extended to multielement mapping of $\mathrm{CP}$ stars and to eclipsing binary systems. Modelling the magnetic field is considerably more demanding: the observations are influenced not only by the field strength, but also by its orientation relative to the line-of-sight which changes as the star rotates. In this paper we present our new magnetic Doppler Imaging code INVERS10 capable of reconstructing the field vector distribution and one additional scalar parameter map of the stellar surface. It was logical to apply this code first to magnetic CP stars where fields are strong and globally organized, and a wealth of observational data already exists.

In the following sections we describe the solution of the radiative transfer for four Stokes parameters, regularization for magnetic DI, minimization algorithm and the implementation of the parallel calculations in INVERS10.

\section{Magnetic Doppler Imaging}

MDI follows the general path of the conventional Doppler Imaging technique (e.g. Piskunov et al. 1990): we search for an optimal fit of synthetic spectra to the sequence of observational data by adjusting the surface distribution of free parameter(s). Free parameters for MDI include the vector map of the magnetic field and, keeping in mind the 
application to magnetic CP stars, the abundance distribution of one chemical element. The observational data consist of a time series of polarization spectra (Stokes $I$, $Q, U$ and $V$ parameters) covering all rotational phases as evenly as possible.

Mathematically MDI is a least-squares minimization problem:

$$
\begin{aligned}
\Psi & =\sum_{\phi \lambda} \omega_{\phi \lambda}^{I}\left[I_{\phi \lambda}^{\mathrm{comp}}(\boldsymbol{B}, Z)-I_{\phi \lambda}^{\mathrm{obs}}\right]^{2} \\
& +\sum_{\phi \lambda} \omega_{\phi \lambda}^{Q}\left[Q_{\phi \lambda}^{\mathrm{comp}}(\boldsymbol{B}, Z)-Q_{\phi \lambda}^{\mathrm{obs}}\right]^{2} \\
& +\sum_{\phi \lambda} \omega_{\phi \lambda}^{U}\left[U_{\phi \lambda}^{\mathrm{comp}}(\boldsymbol{B}, Z)-U_{\phi \lambda}^{\mathrm{obs}}\right]^{2} \\
& +\sum_{\phi \lambda} \omega_{\phi \lambda}^{V}\left[V_{\phi \lambda}^{\mathrm{comp}}(\boldsymbol{B}, Z)-V_{\phi \lambda}^{\mathrm{obs}}\right]^{2} \\
& +\Lambda \cdot R(\boldsymbol{B}, Z) \rightarrow \min
\end{aligned}
$$

where $I, Q, U$ and $V$ are Stokes parameters characterizing polarization status of radiation. They depend on the magnetic field $\boldsymbol{B}$ and abundance distribution $Z$. Weights $\omega$ reflect the relative quality of the data. As in the case of conventional Doppler Imaging, MDI is generally an illposed problem which is solved with the help of regularization (see e.g. Tikhonov 1977) and the corresponding regularization function $R$ scaled by the regularization parameter $\Lambda$ is included in minimization.

Generally, ill-posed problems may be divided in two groups: those with unique and those with multiple solutions. The corresponding role of regularization is different: in the first case it only prevents the numerical procedure from leaving the convergence region due to incompleteness of the data, in the second case it restricts the multitude of solutions with a plausible subset effectively replacing the problem with a well-behaved one. It is important that such regularization would be based on a physical model for the problem.

It is not proven that even conventional DI belongs to the first group, but extensive numerical experiments carried out by several researchers show that solutions converge to the true ones with improved quality and quantity of observations except for degenerate cases (e.g. pole-on or equator-on observations). We see similar behaviour for the case of MDI when a full set of Stokes parameters is used suggesting that MDI may have a unique solution. For the case of $I$ and $V$ observations the problem clearly has no unique solution. Such a situation may arise from the fact that (a) the same $I$ and $V$ can correspond to two different field vectors in the same point on the stellar surface or that (b) two different field distributions result in identical Stokes parameters. Keeping in mind the fact that we have a time series of observations it is easy to show that the first situation can only occur in the degenerate case of $i=$ $90^{\circ}$. Indeed, $I$ and $V$ are sensitive to the field strength and field component oriented along the line-of-sight. For a given surface point with longitude $\phi$ and latitude

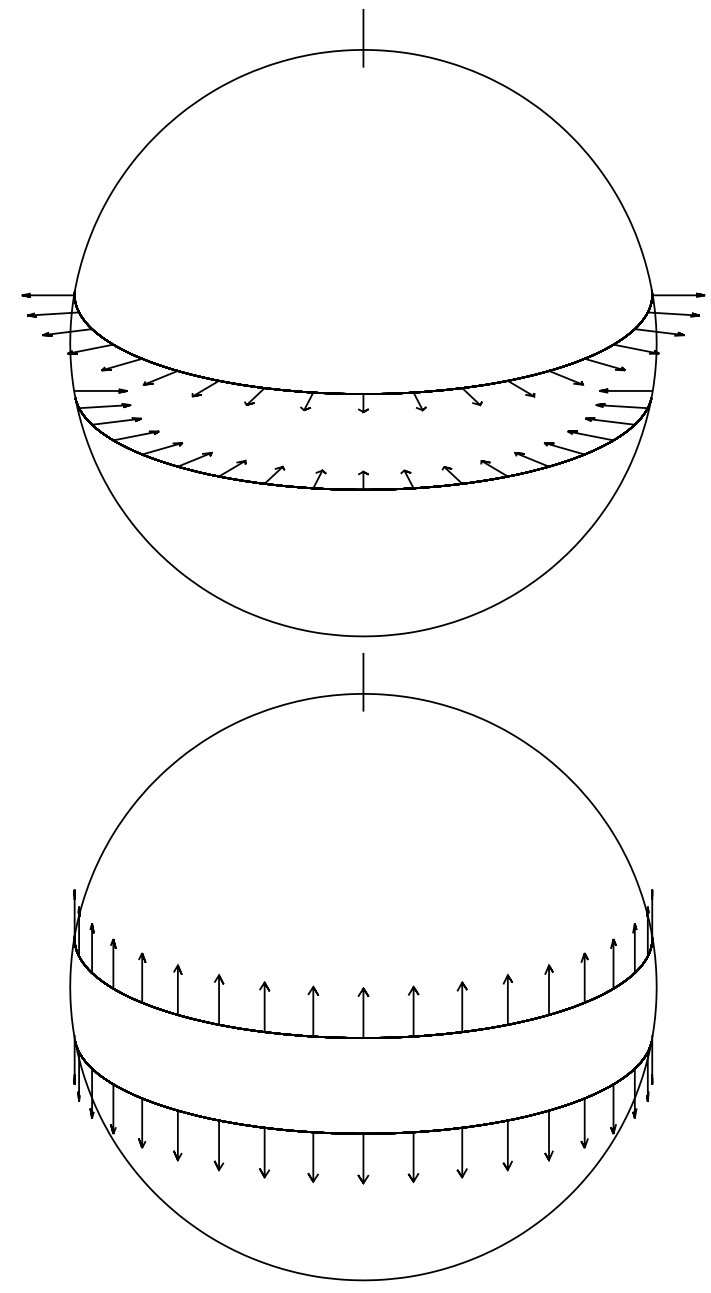

Fig. 1. Two different field distributions resulting in identical time series of $I$ and $V$. In both cases the field vectors are located along the two latitudes $\pm 10^{\circ}$. Top panel: field vectors are parallel to the equatorial plane. Bottom panel: vectors are parallel to the rotational axis. In both cases no time variation is expected and the integration along the lines of constant radial velocities (forming lines parallel to the rotational axis for rigid rotator) results in zero circular polarization. Note, that linear polarization measurements will easily distinguish between the two cases.

$\theta$ (see also Fig. 6) and field defined in the local coordinate system by $\left\{\boldsymbol{B}_{r}, \boldsymbol{B}_{\rho}, \boldsymbol{B}_{\eta}\right\}$ - radial, meridional and azimuthal components, the line-of-sight component of the field will be:

$$
\begin{aligned}
\boldsymbol{B}_{z}= & -\boldsymbol{B}_{\eta} \sin \phi \cos i \\
& -\boldsymbol{B}_{\rho}(\cos \theta \sin i+\sin \theta \cos i \cos \phi) \\
& -\boldsymbol{B}_{r}(\sin \theta \sin i+\cos \theta \cos i \cos \phi) .
\end{aligned}
$$

A degenerate time series means that at least two sets of $\boldsymbol{B}_{r}, \boldsymbol{B}_{\rho}, \boldsymbol{B}_{\eta}$ will satisfy this equation for any $\phi$. Let us take three specific longitudes $\phi=0^{\circ}, 30^{\circ}$ and $60^{\circ}$, then the matrix of the resulting system of linear equations (ignoring the sign) will be:

$$
\begin{array}{lll}
0 & \cos \theta \sin i+\sin \theta \cos i & \sin \theta \sin i-\cos \theta \cos i \\
\frac{1}{2} \cos i & \cos \theta \sin i+\frac{\sqrt{3}}{2} \sin \theta \cos i & \sin \theta \sin i-\frac{\sqrt{3}}{2} \cos \theta \cos i \\
\frac{\sqrt{3}}{2} \cos i & \cos \theta \sin i+\frac{1}{2} \sin \theta \cos i & \sin \theta \sin i-\frac{1}{2} \cos \theta \cos i .
\end{array}
$$


In order to have multiple solutions the determinant must be equal to zero which is only possible if $\cos i=0$. In other words, if we could spatially resolve the stellar surface, time series of circular polarization spectra would be sufficient to reconstruct the 3D structure of the field! Unfortunately, one can easily produce examples of different field distributions resulting in nearly identical (on the level of $10^{-5}$ ) time series of $I$ and $V$ (one of them is shown in Fig. 1) and therefore special, a more restrictive regularization function is required (see Sect. 2.7).

The calculations of the synthetic Stokes parameters consist of the integration of the equation of polarized radiative transfer followed by the disk integration taking into account rotational Doppler shifts. We will consider those two important steps in more detail in the following sections.

\subsection{Synthetic Stokes profiles}

The synthetic Stokes parameters $I, Q, U$ and $V$ are computed by integrating local Stokes vectors at the nodes of a selected surface grid. Often in conventional DI the calculation of the local line profiles is replaced by interpolation in a pre-computed table (see e.g. Piskunov \& Rice 1993). Unfortunately, such an approach cannot be used with MDI as the accurate interpolation of a Stokes vector for a large number of free parameters at each grid point (four in our case) and field-dependent wavelength shifts of the Zeeman components requires a table of unrealistic size. Instead, the equation of magnetic radiative transfer must be solved on-the-fly making the radiative transfer solver (RTS) the core of any MDI code. Such an RTS must be sufficiently accurate to represent the wavelength dependent Stokes parameters radiated from each element on the stellar disk and fast enough to handle large numbers of wavelengths, surface elements and rotational phases involved in MDI.

The polarization status of radiation is fully characterized by the Stokes vector $\boldsymbol{I}=\{I, Q, U, V\}^{\dagger}$. Following the definitions for polarization components given by Rees (1989) we can write the equation of radiative transfer in the magnetic case as a system of four ordinary differential equations for Stokes vector:

$$
\frac{\mathrm{d} \boldsymbol{I}}{\mathrm{d} z}=-\mathcal{K} \boldsymbol{I}+\boldsymbol{J}
$$

where the absorption matrix $\mathcal{K}$ and the emission vector $\boldsymbol{J}$ are given by:

$$
\begin{aligned}
\mathcal{K} & =k_{\mathrm{c}} \mathbb{1}+\sum_{\text {lines }} k_{0}^{\text {line }} \Phi_{\text {line }} \\
\boldsymbol{J} & =k_{\mathrm{c}} S_{\mathrm{c}} e_{0}+\sum_{\text {lines }} k_{0}^{\text {line }} S_{\text {line }} \Phi_{\text {line }} e_{0} .
\end{aligned}
$$

Here $\mathbb{1}$ is a $4 \times 4$ identity matrix, $e_{0}=\{1,0,0,0\}^{\dagger}$, and $k_{\mathrm{c}}$ and $S_{\mathrm{c}}$ are the continuum opacity and source function. The line opacity consists of a sum of the line absorption matrices $\Phi$ multiplied by the line center opacity $k_{0}^{\text {line }}$ for all contributing lines. We assume an unpolarized continuum and LTE throughout the rest of the paper. The polarization of the continuum is irrelevant for an MDI code as it contains little information about surface structures and in all non-degenerate magnetic stars it is small compared to magnetic polarization in spectral lines. For now we also treat line scattering as absorption although the implementation of line scattering is straightforward. The NLTE effects are more difficult to include when dealing with complex blends (although there are several NLTE RTS codes for non-magnetic and even magnetic cases), but here we prefer to restrict ourselves to lines formed in LTE which allows us to set the source functions equal to the Planck function $B_{\lambda}(T)$ and express the emission vector as:

$\boldsymbol{J}_{\lambda}=k_{\mathrm{c}} B_{\lambda}(T) e_{0}+B_{\lambda}(T) \sum_{\text {lines }} k_{0}^{\text {line }} \Phi_{\text {line }} e_{0}$.

Finally we can write the expressions for the absorption matrix $\Phi$ :

$\Phi=\left(\begin{array}{cccc}\phi_{I} & \phi_{Q} & \phi_{U} & \phi_{V} \\ \phi_{Q} & \phi_{I} & \psi_{V} & -\psi_{U} \\ \phi_{U} & -\psi_{V} & \phi_{I} & \psi_{Q} \\ \phi_{V} & \psi_{U} & -\psi_{Q} & \phi_{I}\end{array}\right)$

where:

$$
\begin{aligned}
\phi_{I} & =1 / 2\left(\phi_{p} \sin ^{2} \gamma+1 / 2\left(\phi_{r}+\phi_{b}\right)\left(1+\cos ^{2} \gamma\right)\right) \\
\phi_{Q} & =1 / 2\left(\phi_{p}-1 / 2\left(\phi_{r}+\phi_{b}\right)\right) \sin ^{2} \gamma \cos 2 \chi \\
\phi_{U} & =1 / 2\left(\phi_{p}-1 / 2\left(\phi_{r}+\phi_{b}\right)\right) \sin ^{2} \gamma \sin 2 \chi \\
\phi_{V} & =1 / 2\left(\phi_{r}-\phi_{b}\right) \cos \gamma \\
\psi_{Q} & =1 / 2\left(\psi_{p}-1 / 2\left(\psi_{r}+\psi_{b}\right)\right) \sin ^{2} \gamma \cos 2 \chi \\
\psi_{U} & =1 / 2\left(\psi_{p}-1 / 2\left(\psi_{r}+\psi_{b}\right)\right) \sin ^{2} \gamma \sin 2 \chi \\
\psi_{V} & =1 / 2\left(\psi_{r}-\psi_{b}\right) \cos \gamma
\end{aligned}
$$

The angles $\gamma$ and $\chi$ determine the orientation of the field vector in the observer reference frame as shown in Fig. 2. The $\phi$ 's and the $\psi$ 's in the absorption matrix (6) describe the absorption and anomalous dispersion profiles. For a given transition with magnetic quantum numbers of Zeeman states $M_{\text {lower }}$ and $M_{\text {upper }}$ we denote the type of transition allowed by the selection rule as:

$\Delta M=M_{\text {upper }}-M_{\text {lower }}=\left\{\begin{array}{rl}+1 & \equiv b \\ 0 & \equiv p \\ -1 & \equiv r\end{array}\right.$.

In a moderate magnetic field ( $<10^{6}$ Gauss) assuming LS coupling a level with quantum numbers $L, S$ and $J$ splits into $2 J+1$ states with $M=-J, \ldots, 0, \ldots,+J$ and with Landé factors:

$g=\frac{3}{2}+\frac{S(S+1)-L(L+1)}{2 J(J+1)}$.

For a permitted transition between two Zeeman states the wavelength shift relative to the line center is:

$\Delta \lambda=\frac{e \lambda_{0}^{2}|\boldsymbol{B}|}{4 \pi m c^{2}}\left(g_{\text {lower }} M_{\text {lower }}-g_{\text {upper }} M_{\text {upper }}\right)$. 


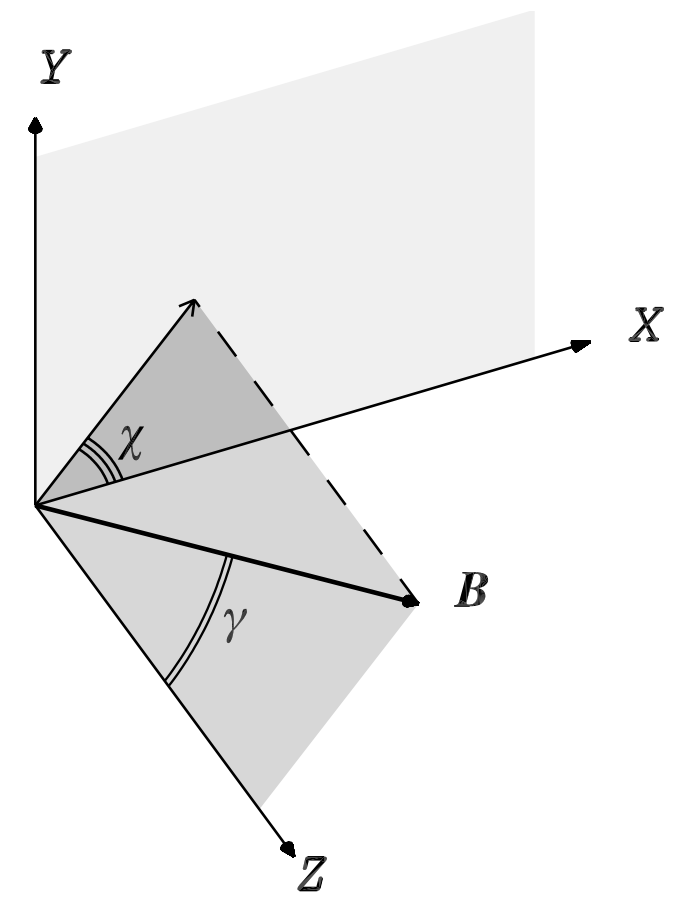

Fig. 2. The orientation of the magnetic field vector in the observer reference frame ( $Z$ points towards the observer) is defined by the angles $\gamma$ and $\chi \cdot \gamma$ is measured from $Z$ to the field vector: $0^{\circ} \leq \gamma \leq 180^{\circ} \cdot \chi$ is measured in the image plane from the $X$-axis counter-clockwise.

For similar Landé factors of the upper and lower level a red shift corresponds to positive $\Delta M$ which explains the convention introduced in (8).

The absorption profiles of $\pi$ and $\sigma_{ \pm}$components:

$$
\begin{aligned}
\phi_{b} & =\sum_{b} A_{b} H\left(a, v-\Delta \lambda_{b} / \Delta \lambda_{\text {Dop }}\right) \\
\phi_{p} & =\sum_{p} A_{p} H\left(a, v-\Delta \lambda_{p} / \Delta \lambda_{\text {Dop }}\right) \\
\phi_{r} & =\sum_{r} A_{r} H\left(a, v-\Delta \lambda_{r} / \Delta \lambda_{\text {Dop }}\right)
\end{aligned}
$$

and their anomalous dispersion profiles:

$$
\begin{aligned}
& \psi_{b}=2 \sum_{b} A_{b} F\left(a, v-\Delta \lambda_{b} / \Delta \lambda_{\text {Dop }}\right) \\
& \psi_{p}=2 \sum_{p} A_{p} F\left(a, v-\Delta \lambda_{p} / \Delta \lambda_{\text {Dop }}\right) \\
& \psi_{r}=2 \sum_{r} A_{r} F\left(a, v-\Delta \lambda_{r} / \Delta \lambda_{\text {Dop }}\right)
\end{aligned}
$$

are well described (in most cases) by the Voigt and Faraday-Voigt functions:

$$
\begin{aligned}
& H(a, v)=\frac{a}{\pi} \int_{-\infty}^{+\infty} \frac{\mathrm{e}^{-y^{2}}}{(v-y)^{2}+a^{2}} \mathrm{~d} y \\
& F(a, v)=\frac{1}{2 \pi} \int_{-\infty}^{+\infty} \frac{(v-y) \mathrm{e}^{-y^{2}}}{(v-y)^{2}+a^{2}} \mathrm{~d} y .
\end{aligned}
$$

Humlíček (1982) provides a fast and accurate approximation for both functions (note that the imaginary part of his approximation corresponds to $2 F$, rather than $F$ ). The normalization of individual Zeeman components is done separately for $b, p$ and $r$ so that: $\sum A_{b}=\sum A_{p}=$ $\sum A_{r}=1$. The Voigt parameters $a$ and $v$ are the Lorentzian line width and the offset from the line center expressed in units of Doppler width:

$$
\begin{aligned}
& a=\frac{\Gamma_{\text {Rad }}+\Gamma_{\text {Stark }}+\Gamma_{\text {van der Waals }}}{4 \pi \Delta \nu_{\text {Dop }}} \\
& v=\left(\lambda-\lambda_{0}\right) / \Delta \lambda_{\text {Dop }} .
\end{aligned}
$$

In order to solve the RT Eq. (2) for a given location on the stellar surface we need to:

- compute the Voigt $a$ and $v$ from Eqs. (15) and (16),

- compute Voigt and Faraday-Voigt functions for each Stokes component,

- compute $\phi$ 's and $\psi$ 's using Eqs. (11) and (12),

- compute the continuous opacity coefficient,

- compute the absorption matrix $\Phi$ from Eqs. (6) and (7) for each contributing spectral line and add them together to form the absorption matrix $\mathcal{K}$ and the emission vector $\boldsymbol{J}$,

throughout the atmosphere. We will not discuss here the calculations of the damping parameters for the case where they are not directly available from a line database like, e.g. VALD (Piskunov et al. 1995), or the calculations of different continuous opacities because INVERS10 inherited these parts from our non-magnetic spectral synthesis code SYNTH (Piskunov 1992).

Once the absorption matrix and the emission vector are prepared we are ready to solve the equation of radiative transfer (2) for each visible surface element using an adaptive wavelength grid. We start by computing Stokes vectors for the center of each Zeeman component and refine the grid until linear interpolation provides the required accuracy.

One of the first codes, capable of solving numerically polarized radiative transfer equation, was created by Landi Degl'Innocenti (1976), but the Runge-Kutta integrator used there is too slow for our purpose. Instead we have considered three types of more efficient RTS for INVERS10: Feautrier, Diagonal Element Lambda Operator (DELO) and Hermitian methods.

\subsection{Feautrier RTS}

The Feautrier method is well established as the most stable and fast long characteristic RTS for the non-magnetic case. Therefore, it was logical to extend it to the case with a magnetic field. Such an algorithm was first formulated by Auer et al. (1977) (AHH). Here we repeat basic math from the seminal AHH paper to help us compare the different RTS.

As in the conventional (non-magnetic) case the intensity is split into two flows: $I^{+}$for radiation propagating towards the surface of the star and $I^{-}$for radiation directed inwards. Next, after introducing the two new variables $P=\left(I^{+}+I^{-}\right) / 2$ and $R=\left(I^{+}-I^{-}\right) / 2$ the equation 
of radiative transfer is replaced by a second order differential equation for $P$.

The same principle is generalized to the magnetic case as following. Let $\boldsymbol{I}^{+}$be the Stokes vector characterizing the radiation propagating out of the atmosphere while $\boldsymbol{I}^{-}$ corresponds to the radiation in the opposite direction. Note that for $\boldsymbol{I}^{-}$we also have to switch the $b$ and the $r$ Stokes components as the definition of the left and right circular polarization is direction dependent (e.g. Rees \& Murphy 1987). The switch of direction corresponds to the change of angles to $180^{\circ}-\gamma$ and to $-\chi$. Therefore, the line absorption matrix for $\boldsymbol{I}^{+}$is related to the matrix for $\boldsymbol{I}^{-}$:

$\Phi^{+}=\left(\begin{array}{cccc}\phi_{I} & \phi_{Q} & \phi_{U} & \phi_{V} \\ \phi_{Q} & \phi_{I} & \psi_{V} & -\psi_{U} \\ \phi_{U} & -\psi_{V} & \phi_{I} & \psi_{Q} \\ \phi_{V} & \psi_{U} & -\psi_{Q} & \phi_{I}\end{array}\right)$

$\Phi^{-}=\left(\begin{array}{rrrr}\phi_{I} & \phi_{Q} & -\phi_{U} & \phi_{V} \\ \phi_{Q} & \phi_{I} & -\psi_{V} & -\psi_{U} \\ -\phi_{U} & \psi_{V} & \phi_{I} & -\psi_{Q} \\ \phi_{V} & \psi_{U} & \psi_{Q} & \phi_{I}\end{array}\right)$

This follows from Eqs. (6) and (7) and from the fact that the Voigt function (Eq. (13)) is symmetric while the Faraday-Voigt function is anti-symmetric (Eq. (14)), leading to an additional sign change for all $\psi^{-}$but not for $\phi^{-}$. Now we note that $\Phi^{-}$can be made identical to $\Phi^{+}$if we define $\boldsymbol{I}^{-}$as $\{I, Q,-U, V\} \dagger$. For the new definition of the radiation flows we can write two identical radiative transfer equations:

$\pm \frac{\mathrm{d} \boldsymbol{I}^{ \pm}}{\mathrm{d} z}=-\mathcal{K} \boldsymbol{I}^{ \pm}+\boldsymbol{J}$

and after adding and subtracting them and introducing the Feautrier variables $\boldsymbol{P}=\left(\boldsymbol{I}^{+}+\boldsymbol{I}^{-}\right) / 2$ and $\boldsymbol{R}=\left(\boldsymbol{I}^{+}{ }_{-}\right.$ $\left.\boldsymbol{I}^{-}\right) / 2$ we get the familiar equations:

$$
\begin{aligned}
\frac{\mathrm{d} \boldsymbol{P}}{\mathrm{d} z} & =-\mathcal{K} \boldsymbol{R} \\
\frac{\mathrm{d} \boldsymbol{R}}{\mathrm{d} z} & =-\mathcal{K} \boldsymbol{P}+\boldsymbol{J} .
\end{aligned}
$$

We combine them into a single 2 nd order equation for $\boldsymbol{P}$ :

$\frac{\mathrm{d}}{\mathrm{d} z}\left(\mathcal{K}^{-1} \frac{\mathrm{d} \boldsymbol{P}}{\mathrm{d} z}\right)=\mathcal{K} \boldsymbol{P}-\boldsymbol{J}$.

Boundary conditions are set on both ends of the integration path:

$\mathcal{K}^{-1} \frac{\mathrm{d} \boldsymbol{P}}{\mathrm{d} z}=-\boldsymbol{P}$

at the surface,

$\mathcal{K}^{-1} \frac{\mathrm{d} \boldsymbol{P}}{\mathrm{d} z}=\boldsymbol{P}-\boldsymbol{I}^{+}$

at maximum $z$.

We follow Auer et al. (1977) in using a Taylor expansion to improve the approximation at the inner boundary:

$\boldsymbol{I}^{+}=\left(B_{\lambda}-\mathcal{K}^{-1} \frac{\mathrm{d} B_{\lambda}}{\mathrm{d} z}\right) e_{0}$
At this point, we note that we can easily switch from the simple geometrical depth scale along the line-of-sight $z$ to the depth scale perpendicular to the surface $z^{\prime}$ by introducing $\mu$ - the cosine of the angle between $z$ and $z^{\prime}$. Furthermore, model atmospheres (e.g. Kurucz grid) are often computed on a scale of column mass rather than geometrical depth. We can convert to this scale by replacing $k_{\mathrm{c}}$ and $k_{0}^{\text {line }}$ computed per unit of length, with opacities computed per unit of mass and substituting $\rho \mathrm{d} z^{\prime}$ with $-\mathrm{d} m$.

$$
\begin{aligned}
\mu^{2} \frac{\mathrm{d}}{\mathrm{d} m}\left(\mathcal{K}^{-1} \frac{\mathrm{d} \boldsymbol{P}}{\mathrm{d} m}\right) & =\mathcal{K} \boldsymbol{P}-\boldsymbol{J} \\
\mathcal{K}^{-1} \frac{\mathrm{d} \boldsymbol{P}}{\mathrm{d} m} & =-\left.\boldsymbol{P}\right|_{m_{0}} \\
\mathcal{K}^{-1} \frac{\mathrm{d} \boldsymbol{P}}{\mathrm{d} m} & =\boldsymbol{P}-\left.e_{0}\left(B_{\lambda}-\mathcal{K}^{-1} \frac{\mathrm{d} B_{\lambda}}{\mathrm{d} m}\right)\right|_{m_{N}} .
\end{aligned}
$$

On a discrete grid the Eqs. (23) can be represented with finite differences. For depth points $i=0,1, \ldots, N-1$ we define the quantities:

$\delta_{i}=\left(m_{i+1}-m_{i}\right) / \mu$ and $\Delta_{i}=\frac{1}{2}\left(\mathcal{K}_{i+1}^{-1}+\mathcal{K}_{i}^{-1}\right) \delta_{i}$.

Then

$\left(\begin{array}{cclll}\mathcal{B}_{1} & -\mathcal{C}_{1} & \ldots & 0 & 0 \\ -\mathcal{A}_{1} & \mathcal{B}_{2} & \ldots & 0 & 0 \\ 0 & -\mathcal{A}_{2} & \ldots & 0 & 0 \\ \ldots & \ldots & \ldots & \ldots \\ 0 & 0 & \ldots & \mathcal{B}_{N-1} & -\mathcal{C}_{N-1} \\ 0 & 0 & \ldots & -\mathcal{A}_{N-1} & \mathcal{B}_{N}\end{array}\right) \cdot\left(\begin{array}{l}\boldsymbol{P}_{1} \\ \boldsymbol{P}_{2} \\ \boldsymbol{P}_{3} \\ \ldots \\ \boldsymbol{P}_{N-1} \\ \boldsymbol{P}_{N}\end{array}\right)=\left(\begin{array}{l}\boldsymbol{D}_{1} \\ \boldsymbol{D}_{2} \\ \boldsymbol{D}_{3} \\ \ldots \\ \boldsymbol{D}_{N-1} \\ \boldsymbol{D}_{N}\end{array}\right)$

where $\mathcal{A}_{i}, \mathcal{B}_{i}$ and $\mathcal{C}_{i}$ are $4 \times 4$ matrices, and $\boldsymbol{D}_{i}$ and $\boldsymbol{P}_{i}$ are 4 element vectors. The first and the last row are given by the boundary conditions:

$\mathcal{B}_{1}=\Delta_{1} / \delta_{1}+1 / \delta_{1}+\mathcal{K}_{1}$

$\mathcal{C}_{1}=\Delta_{1} / \delta_{1}$

$\boldsymbol{D}_{1}=\boldsymbol{J}_{1}$

$\mathcal{A}_{N}=\Delta_{N-1}-\frac{1}{2} 1$

$\mathcal{B}_{N}=\Delta_{N-1}+\frac{1}{2} 1$

$\boldsymbol{D}_{N}=\frac{1}{2}\left(B_{\lambda N}+B_{\lambda N-1}\right) e_{0}+$ $\Delta_{N-1}\left(B_{\lambda N}-B_{\lambda N-1}\right) e_{0}$

while the second order equation couples 3 diagonals:

$\begin{aligned} \mathcal{A}_{i} & =2 \Delta_{i-1} /\left(\delta_{i}+\delta_{i-1}\right) \\ \mathcal{C}_{i} & =2 \Delta_{i} /\left(\delta_{i}+\delta_{i-1}\right) \\ \mathcal{B}_{i} & =\mathcal{A}_{i}+\mathcal{C}_{i}+\mathcal{K}_{i} .\end{aligned}$

The right hand side for the intermediate rows is simply the emission vector $\boldsymbol{J}_{i}$ given by Eq. (5). We note that the 
whole set of equations (25)-(26) is a block tri-diagonal linear system that can be solved with a conventional forward and backward elimination procedure. First, we get rid of the lower diagonal elements ( $\mathcal{A}$ 's):

$\mathcal{C}_{1}^{\prime}=\mathcal{B}_{1}^{-1} \mathcal{C}_{1}$

$\boldsymbol{D}_{1}^{\prime}=\mathcal{B}_{1}^{-1} \boldsymbol{D}_{1}$

$\mathcal{C}_{i}^{\prime}=\left(\mathcal{B}_{i}-\mathcal{A}_{i-1} \mathcal{C}_{i-1}^{\prime}\right)^{-1} \mathcal{C}_{i}$

$\boldsymbol{D}_{i}^{\prime}=\left(\mathcal{B}_{i}-\mathcal{A}_{i-1} \mathcal{C}_{i-1}^{\prime}\right)^{-1}\left(\boldsymbol{D}_{i}+\mathcal{A}_{i-1} \boldsymbol{D}_{i-1}^{\prime}\right)$

and then perform the back substitution to obtain vectors $\boldsymbol{P}$ :

$\boldsymbol{P}_{N}=\boldsymbol{D}_{N}^{\prime}$

$\boldsymbol{P}_{i}=\boldsymbol{D}_{i}^{\prime}+\mathcal{C}_{i}^{\prime} \boldsymbol{P}_{i+1}$.

The emerging Stokes vector $\left.\boldsymbol{I}\right|_{z=0}=\left.\boldsymbol{I}^{+}\right|_{z=0}=2 \boldsymbol{P}_{1}$.

The method is accurate and efficient as in the nonmagnetic case but requires two matrix inversions per depth point and at least one of them needs pivoting to avoid numerical instability while solving the block tridiagonal system in the case of strong lines and a substantial field (Piskunov 1999).

The Feautrier method gives accurate results even on a rather sparse grid. This is particularly useful in the case where grid refinement is difficult (e.g. multidimensional RT) or when we are interested to know the accurate radiation field throughout the whole atmosphere. Spectral synthesis of polarized radiation in INVERS10 only requires the knowledge of the emergent Stokes parameters along the rays directed towards the observer, therefore we also consider short characteristic methods.

\subsection{DELO RTS}

The Diagonal Element Lambda Iteration method has been suggested by Rees et al. (1989) who noted that the absorption matrix $\mathcal{K}$ is dominated by the main diagonal which consists of identical elements for each spectral line. Let us consider first a case of an isolated line. Then the diagonal elements of $\mathcal{K}$ given by the Eq. (6) are $k_{\mathrm{c}}+k_{0}^{\text {line }} \phi_{I}$. Introducing a modified absorption matrix and the emission vector:

$\mathcal{K}^{\prime}=\mathcal{K} / k^{\text {tot }}-1$

$\boldsymbol{S}^{\prime}=\boldsymbol{J} / k^{\mathrm{tot}}$

where $k^{\text {tot }}=k_{\mathrm{c}}+k_{0}^{\text {line }} \phi_{I}$ and defining optical depth as $\mathrm{d} \tau=-k^{\text {tot }} \mathrm{d} z$ we can re-write the radiative transfer equation in the form:

$\frac{\mathrm{d} \boldsymbol{I}}{\mathrm{d} \tau}=\boldsymbol{I}-\left(\boldsymbol{S}^{\prime}-\mathcal{K}^{\prime} \boldsymbol{I}\right)=\boldsymbol{I}-\boldsymbol{S}$.

The new source function vector $\boldsymbol{S}=\left(\boldsymbol{S}^{\prime}-\mathcal{K}^{\prime} \boldsymbol{I}\right)$ is not very different from $S^{\prime}$ because $\mathcal{K}^{\prime}$ is small. For a given depth interval between points $\tau_{i}$ and $\tau_{i+1}$ the formal solution of Eq. (31) can be written as:

$\boldsymbol{I}\left(\tau_{i}\right)=\epsilon_{i} \boldsymbol{I}\left(\tau_{i+1}\right)+\int_{\tau_{i}}^{\tau_{i+1}} \mathrm{e}^{-\left(\tau-\tau_{i}\right)} \boldsymbol{S}(\tau) \mathrm{d} \tau$

where:

$\epsilon_{i}=\mathrm{e}^{-\delta_{i}}$ and $\delta_{i}=\tau_{i+1}-\tau_{i}$.

Equation (32) looks very similar to a formal solution of a non-magnetic radiative transfer equation. Although $\boldsymbol{S}$ depends on the Stokes vector, for a sufficiently dense depth grid the source vector can be well approximated with a linear function:

$\boldsymbol{S}(\tau)=\left[\left(\tau_{i+1}-\tau\right) \boldsymbol{S}_{i}+\left(\tau-\tau_{i}\right) \boldsymbol{S}_{i+1}\right] / \delta_{i}$

and substituting this expression into Eq. (32) we can perform the integral analytically (see Eqs. (62)-(67) in Rees et al. 1989). This gives us a linear relation between Stokes vectors in points $i+1$ and $i$ :

$\mathcal{X}_{i} \cdot \boldsymbol{I}\left(\tau_{i}\right)=\mathcal{Y}_{i} \cdot \boldsymbol{I}\left(\tau_{i+1}\right)+\mathcal{Z}_{i}$

where:

$\mathcal{X}_{i}=11+\left(\alpha_{i}-\beta_{i}\right) \mathcal{K}_{i}^{\prime}$

$\mathcal{Y}_{i}=\left(\epsilon_{i} \mathbb{1}-\beta_{i} \mathcal{K}_{i+1}^{\prime}\right)$

$\mathcal{Z}_{i}=\left(\alpha_{i}-\beta_{i}\right) \boldsymbol{S}_{i}^{\prime}+\beta_{i} \boldsymbol{S}_{i+1}^{\prime}$

$\alpha_{i}=1-\epsilon_{i}$

$\beta_{i}=\left[1-\left(1+\delta_{i}\right) \epsilon_{i}\right] / \delta_{i}$.

The boundary condition for Eq. (32) is set deep in the atmosphere. The corresponding asymptotic approximation is readily given by Eq. (22). The main advantage of the DELO method is that it requires only one pass through the atmosphere. Equations (36) can be generalized to the case of multiple blended lines and column mass as a depth parameter. Before doing it, we note that in case of a blend the diagonal elements of $\mathcal{K}$ will be $k_{\mathrm{c}}+\sum k_{0}^{\text {line }} \phi_{I}^{\text {line }}$ so the new definition for $k^{\text {tot }}$ should be:

$k^{\text {tot }}=k_{\mathrm{c}}+\sum_{\text {lines }} k_{0}^{\text {line }} \phi_{I}^{\text {line }}$.

We also note that for the mass column as an independent depth parameter $\delta_{i} \approx \frac{1}{2}\left(k_{i}^{\text {tot }}+k_{i+1}^{\text {tot }}\right)\left(m_{i+1}-m_{i}\right)$. The rest of the derivation of Eqs. (35) and (36) holds directly. The integration starts from the deep layers where the initial Stokes vector is given by Eq. (22), then for each step we evaluate matrices $\mathcal{X}, \mathcal{Y}$ and vector $\mathcal{Z}$ using Eqs. (36) and compute the Stokes vector at point $i$ from Eq. (35). Note that instead of a matrix inversion at each step we solve a system of linear equations which is numerically stable because for small $\delta_{i}$ the factor $\left(\alpha_{i}-\beta_{i}\right)$ is of the order of $\delta_{i} / 2$ and $\mathcal{X}_{i}$ is dominated by its main diagonal. Therefore, no pivoting is required.

The accuracy of the DELO method primarily depends on the accuracy of the linear approximation for $\boldsymbol{S}$ given by Eq. (34). Higher precision for the emerging Stokes vector 
can be obtained by refining the depth grid and/or using a higher order approximation for the source function instead of Eq. (34). DELO algorithm based on quadratic approximation for the source function was suggested by Socas-Navarro et al. (2000). Equation (34) for the interval $\left(\tau_{i}, \tau_{i+1}\right)$ is replaced with:

$\boldsymbol{S}(\tau)=\alpha_{i} \boldsymbol{S}_{i}+\beta_{i} \boldsymbol{S}_{i+1}+\gamma_{i} \boldsymbol{S}_{i+2}$

where:

$$
\begin{aligned}
\alpha_{i} & =\frac{\boldsymbol{z}-\delta_{i+1} \boldsymbol{y}}{\left(\delta_{i}+\delta_{i+1}\right) \delta_{i}} \\
\beta_{i} & =\frac{\left(\delta_{i+1}+\delta_{i}\right) \boldsymbol{y}-\boldsymbol{z}}{\delta_{i} \delta_{i+1}} \\
\gamma_{i} & =\mathbf{x}+\frac{\boldsymbol{z}-\left(\delta_{i}+2 \delta_{i+1}\right) \boldsymbol{y}}{\delta_{i+1}\left(\delta_{i}+\delta_{i+1}\right)} \\
\boldsymbol{x} & =1-\epsilon_{i} \\
\boldsymbol{y} & =\delta_{i}-\boldsymbol{x} \\
\boldsymbol{z} & =\delta_{i}^{2}-2 \boldsymbol{y}
\end{aligned}
$$

The overhead for the quadratic version of DELO is negligible compared to the linear version. We conclude that DELO algorithm is fast, robust and sufficiently accurate to be considered for INVERS10.

\subsection{Hermitian RTS}

The Hermitian RTS suggested by Ruiz Cobo et al. (1999) is based on an excellent idea to use the 4th order Taylor expansion in point $i+1$ in the atmosphere to estimate the value of the Stokes vector at the point $i$ (points are numbered $1, \ldots, N$ starting at the surface) while the corresponding derivatives are computed from the moments of the radiative transfer equation. Indeed, the 4 th order Taylor expansion for the Stokes vector is given by:

$\boldsymbol{I}\left(m_{i}\right)=\boldsymbol{I}\left(m_{i+1}\right)+\left.\sum_{n=1}^{4} \frac{\delta_{i}^{n}}{n !} \frac{\mathrm{d}^{n} \boldsymbol{I}}{\mathrm{d} m^{n}}\right|_{m_{i+1}}$

where $\delta_{i}=m_{i+1}-m_{i}$. We can also take the first and the second derivative of $\boldsymbol{I}$ in point $m_{i}$ using Eq. (39):

$$
\begin{aligned}
\boldsymbol{I}^{\prime}\left(m_{i}\right) & =\frac{\mathrm{d} \boldsymbol{I}}{\mathrm{d} m}+\delta_{i} \frac{\mathrm{d}^{2} \boldsymbol{I}}{\mathrm{d} m^{2}}+\frac{1}{2} \delta_{i}^{2} \frac{\mathrm{d}^{3} \boldsymbol{I}}{\mathrm{d} m^{3}}+\left.\frac{1}{6} \delta_{i}^{3} \frac{\mathrm{d}^{4} \boldsymbol{I}}{\mathrm{d} m^{4}}\right|_{m_{i+1}} \\
\boldsymbol{I}^{\prime \prime}\left(m_{i}\right) & =\frac{\mathrm{d}^{2} \boldsymbol{I}}{\mathrm{d} m^{2}}+\delta_{i} \frac{\mathrm{d}^{3} \boldsymbol{I}}{\mathrm{d} m^{3}}+\left.\frac{1}{2} \delta_{i}^{2} \frac{\mathrm{d}^{4} \boldsymbol{I}}{\mathrm{d} m^{4}}\right|_{m_{i+1}} .
\end{aligned}
$$

Now we can eliminate the 3 rd and the 4 th derivatives in Eq. (39) by adding to it Eq. (40) multiplied by $\delta_{i} / 2$ and Eq. (41) multiplied by $\delta_{i}^{2} / 12$. The resulting expression is:

$$
\begin{aligned}
\boldsymbol{I}\left(m_{i}\right)= & \boldsymbol{I}\left(m_{i+1}\right)+\frac{\delta}{2}\left[\boldsymbol{I}^{\prime}\left(m_{i+1}\right)+\boldsymbol{I}^{\prime}\left(m_{i}\right)\right] \\
& +\frac{\delta^{2}}{12}\left[\boldsymbol{I}^{\prime \prime}\left(m_{i+1}\right)-\boldsymbol{I}^{\prime \prime}\left(m_{i}\right)\right]
\end{aligned}
$$

We obtained a 4th order implicit scheme that connects the Stokes vector and its first and second derivatives in two adjacent points. It should be noted that all transformations leading to Eq. (42) are precise - no finite difference approximation has been made so far! The derivatives of the Stokes vector are expressed from the transfer Eq. (2). Note also that we are using column mass as depth parameter for consistency with other algorithms, therefore, the sign of the right hand side is opposite to that in Ruiz Cobo et al. 1999):

$$
\begin{aligned}
\boldsymbol{I}^{\prime}(m) & =\mathcal{K} \boldsymbol{I}-\mathcal{K} \boldsymbol{S} \\
\boldsymbol{I}^{\prime \prime}(m) & =\mathcal{K}^{\prime}(\boldsymbol{I}-\boldsymbol{S})+\mathcal{K}\left(\boldsymbol{I}^{\prime}-\boldsymbol{S}^{\prime}\right) \\
& =\mathcal{K}^{\prime}(\boldsymbol{I}-\boldsymbol{S})+\mathcal{K}\left(\mathcal{K} \boldsymbol{I}-\mathcal{K} \boldsymbol{S}-\boldsymbol{S}^{\prime}\right) .
\end{aligned}
$$

Substituting Eqs. (43) and (44) into Eq. (42) we get the recursive relation for the Hermitian scheme in form of a system of linear equations:

$\mathcal{U}_{i} \boldsymbol{I}_{i}=\mathcal{V}_{i+1} \boldsymbol{I}_{i+1}-\mathcal{W}_{i, i+1}$

where indices refer to the depth points and:

$$
\begin{aligned}
\mathcal{U}_{i}=11- & \frac{\delta_{i}}{2} \mathcal{K}_{i}+\frac{\delta_{i}}{12}\left[\mathcal{K}_{i}^{\prime}+\mathcal{K}_{i} \mathcal{K}_{i}\right] \\
\mathcal{V}_{i+1}=11 & +\frac{\delta_{i}}{2} \mathcal{K}_{i+1}+\frac{\delta_{i}}{12}\left[\mathcal{K}_{i+1}^{\prime}+\mathcal{K}_{i+1} \mathcal{K}_{i+1}\right] \\
\mathcal{W}_{i, i+1}= & \frac{\delta_{i}}{2}\left[\mathcal{K}_{i} \boldsymbol{S}_{i}+\mathcal{K}_{i+1} \boldsymbol{S}_{i+1}\right]- \\
& -\frac{\delta_{i}^{2}}{12}\left[\mathcal{K}_{i}^{\prime} \boldsymbol{S}_{i}+\mathcal{K}_{i} \mathcal{K}_{i} \boldsymbol{S}_{i}+\mathcal{K}_{i} \boldsymbol{S}_{i}^{\prime}-\right. \\
& \left.-\mathcal{K}_{i+1}^{\prime} \boldsymbol{S}_{i+1}-\mathcal{K}_{i+1} \mathcal{K}_{i+1} \boldsymbol{S}_{i+1}-\mathcal{K}_{i+1} \boldsymbol{S}_{i+1}^{\prime}\right]
\end{aligned}
$$

The boundary condition is set deep in the atmosphere in the form given by Eq. (22).

The algorithm appears to be efficient and robust but the dependence of convergence on the quality of approximation for derivatives needs to be investigated. Ruiz Cobo et al. suggest using a piecewise parabolic approximation for the source vector and the absorption matrix. Our experience is that an insufficiently accurate approximation for the derivatives, in particular in optically thick regions, results in very slow convergence.

\subsection{Comparison of the three RTS}

The important properties of the RTS are the accuracy (precision on a given grid) and convergence (precision improvement with grid refinement). In order to study the properties of the RTS described in the previous sections as function of depth and line strength we compute Stokes profiles for two models: solar $\left(T_{\text {eff }}=5770 \mathrm{~K}, \log g=4.44\right)$ and an A-star $\left(T_{\text {eff }}=9000 \mathrm{~K}, \log g=4\right)$ in the $4845 \AA$ and $4590 \AA$ regions. Figure 4 shows the accuracy through the atmosphere of an A-star (disk center) for non-polarized continuum using a grid with 10 points-per-decade of the optical depth. (Depending on the wavelength region the standard model atmospheres of Kurucz (1993) typically contain 8-9 points per decade.) The reference calculation is done with a Runge-Kutta algorithm. Both Feautrier and 

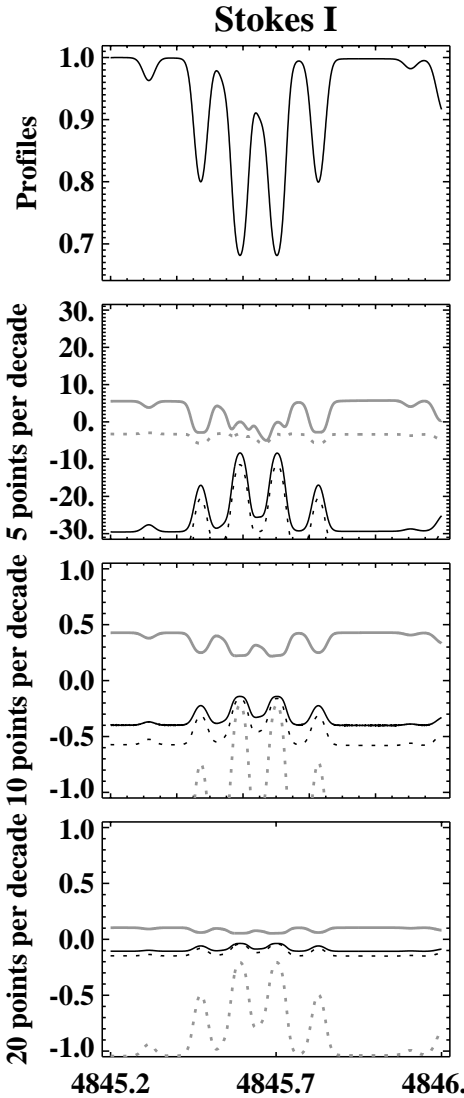
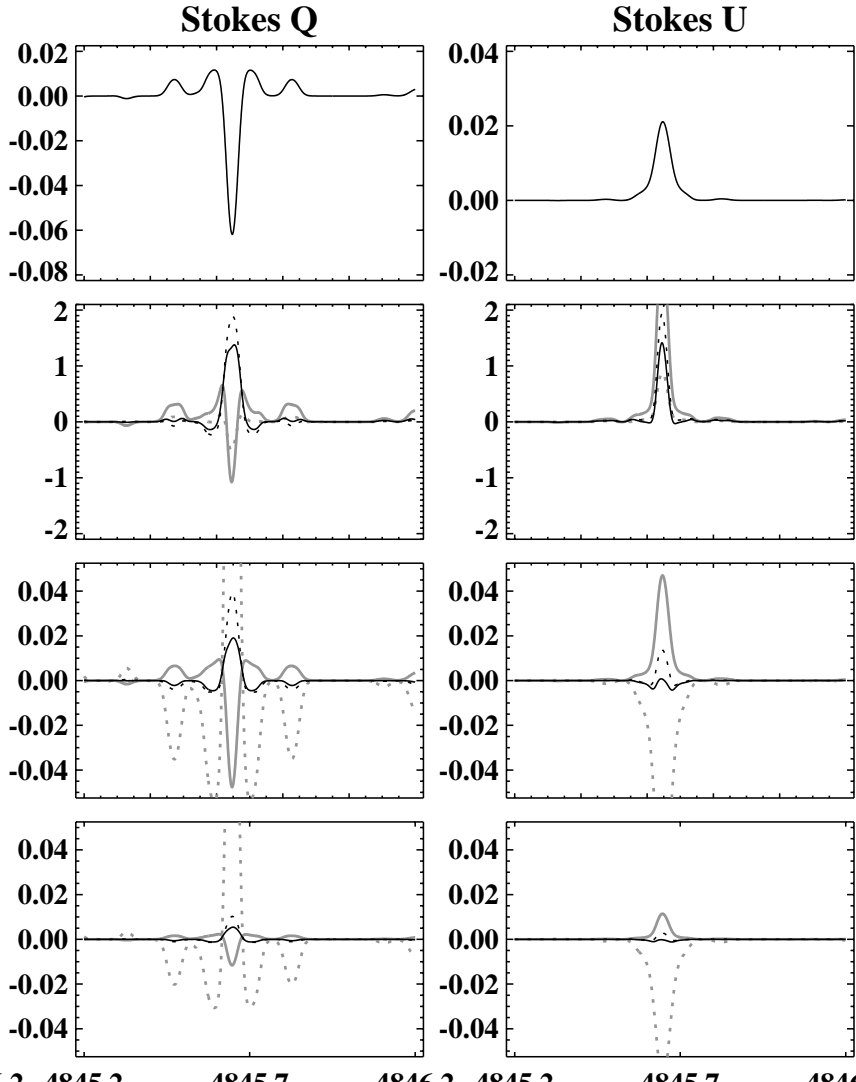
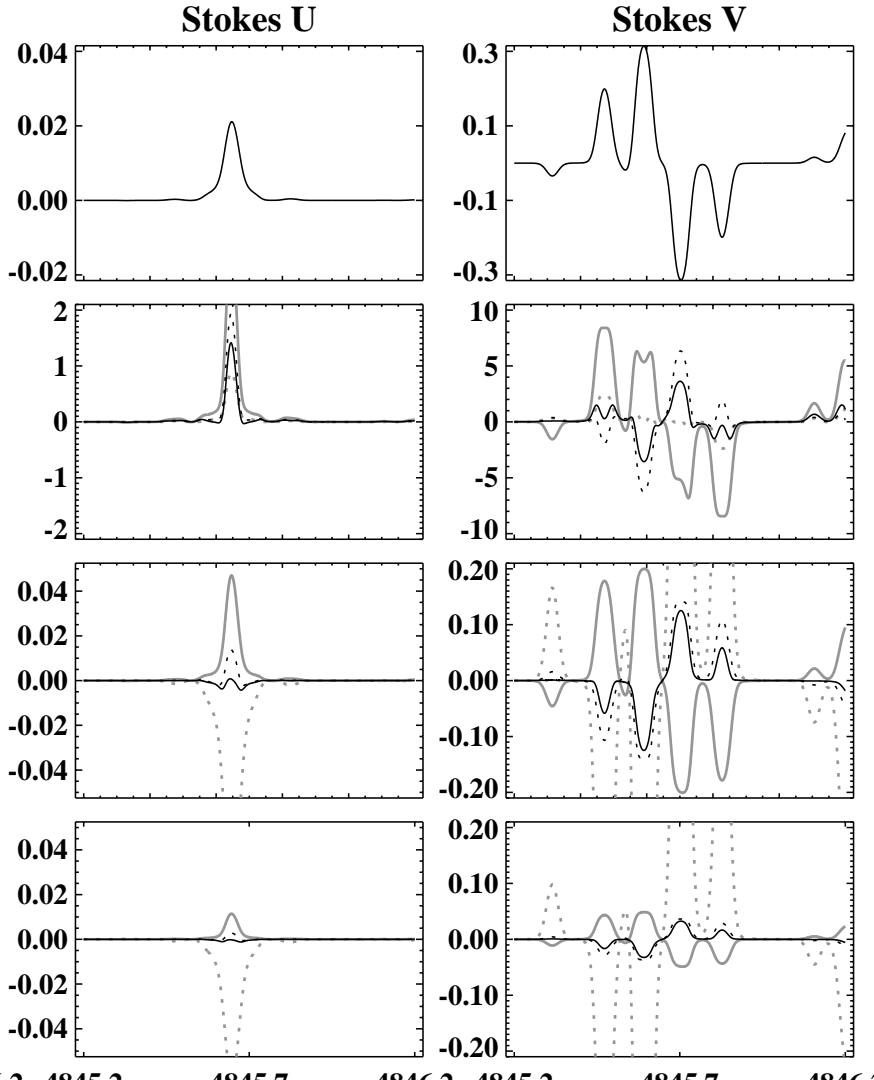

46.24845 .2

Fig. 3. Comparison of the four RTSs as a function of grid density and wavelength. Solid grey line - Feautrier, dashed grey line - Hermitian, solid black line - quadratic DELO and the dashed black line - linear DELO. The three lower rows of plots show the differences in percent of the corresponding Stokes profiles from the Feautrier calculations with 200 points-per-decade normalized to the continuum level.

DELO achieved an accuracy of $0.1 \%$ with quadratic DELO showing the best result. The problem with the Hermitian algorithm is clearly related to the interpolation at the large optical depths. Figure 3 compares the performance of the algorithms as function of wavelength and grid density for the solar model. The line list has been obtained from VALD (Kupka et al. 1999). Spectra were computed for a small offset from the disk center $(\mu=0.97)$ and a $10 \mathrm{kGauss}$ radial field. The reference Stokes profiles have been computed with the Feautrier algorithm on a very dense grid (200 points-per-decade). The results confirm the high accuracy of the Feautrier algorithm even for a sparse grid. For dense grids DELO shows similar accuracy. Quadratic DELO is noticeably better than linear for intermediate grids. For denser grids as expected the results for both versions are very similar. The Hermitian algorithm suffers from poor convergence.

Table 1 compares the performance of all four methods on different computer platforms. For a typical MDI problem the local Stokes profiles are computed using model atmospheres with 8-12 points-per-decade. Considering the the results of the comparison we have incorporated both the Feautrier and DELO algorithms in the INVERS10 program.
Table 1. Computing time for 70000 wavelength points and one spectral line with Zeeman triplet pattern.

\begin{tabular}{lcc}
\hline Algorithm & $\begin{array}{c}\text { HP PA8000 } \\
440 \mathrm{MHz}\end{array}$ & $\begin{array}{c}\text { Intel Pentium III } \\
800 \mathrm{MHz}\end{array}$ \\
\hline Feautrier & $60.1 \mathrm{~s}$ & $59.0 \mathrm{~s}$ \\
DELO (linear) & $17.4 \mathrm{~s}$ & $24.8 \mathrm{~s}$ \\
DELO (quadratic) & $17.7 \mathrm{~s}$ & $23.0 \mathrm{~s}$ \\
Hermitian & $46.5 \mathrm{~s}$ & $40.9 \mathrm{~s}$ \\
\hline
\end{tabular}

\subsection{Disk integration}

Following Vogt et al. (1987), we divide the stellar surface into $N$ approximately equal area zones. With such a division the zones at high latitudes cover a larger range in longitude than those near the equator. Figure 5 shows an example of zonal division into 695 surface elements that was used for our test calculations.

Contribution of a surface zone to the disk-integrated spectrum depends on the projected area of a zone, limb angle and Doppler shift due to stellar rotation. These quantities must be calculated for each surface element at each rotational phase. Since these parameters do not change during the inversion procedure, INVERS10 calculates them 


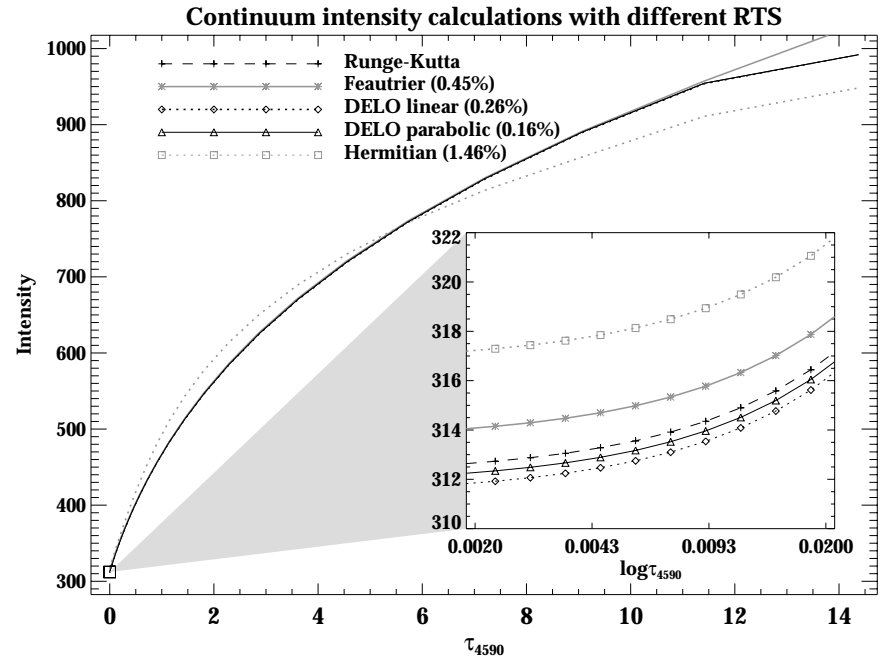

Fig. 4. The accuracy of the four RTSs for continuum intensity as a function of depth. Grid density is 10 points-per-decade of optical depth. The results are marginally different at the surface. The Hermitian algorithm has difficulties at large optical depths.

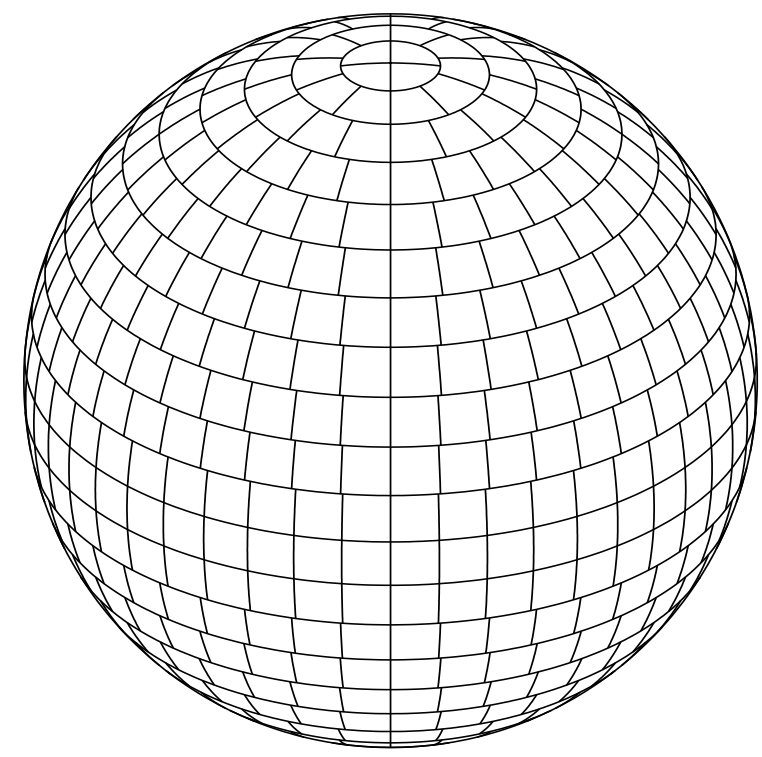

Fig. 5. Subdivision of the stellar surface adopted for disk integration. The surface is divided into 23 latitude belts, and the total number of surface zones is 695 . At this inclination of $60^{\circ}$, the visible hemisphere is covered by 378 zones.

only once, before evaluation of local Stokes vectors. Figure 5 shows, that due to the complex projected shapes of surface elements, the mean rotational Doppler shift and limb angle of a zone do not generally coincide with the values calculated for its geometrical center. In order to achieve high accuracy of disk integration independently of the grid density we subdivide each zone into a number of small $\left(1^{\circ} \times 1^{\circ}\right)$ surface elements. Doppler shift, limb angle and projected area are evaluated for each such element and then the average values of these quantities are constructed for the visible part of a zone. We also compute the range of radial velocities within each surface zone.

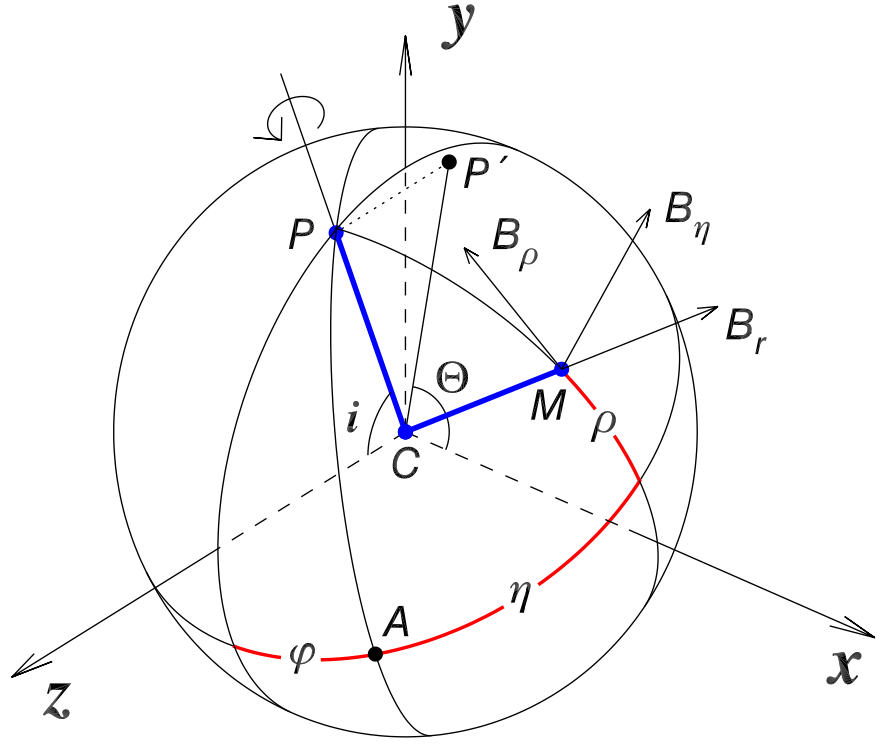

Fig. 6. Stellar and observer coordinate systems. Explanation of the figure is given in the text.

Formulation of the polarized radiative transfer equation also requires evaluation of the magnetic vector components in the observer coordinate system for each surface element at each rotational phase. Definitions of stellar and observer coordinate systems implemented in INVERS10 are illustrated in Fig. 6.

The stellar reference frame is selected in such a way, that at zero inclination the observer is looking at the stellar pole $P$ with zero longitude $P A$ aligned with the $y$ axis and longitudes $\eta$ increasing counter-clockwise. The star rotates in a counter-clockwise direction around the axis $C P$, with phase angle $\varphi$ counted from the $y z$ plane towards zero longitude $P A$. Latitudes $\rho$ are assumed to be positive above and negative below the stellar rotational equator. At any surface point $M$ the magnetic vector $\boldsymbol{B}$ is represented by a combination of radial $B_{\mathrm{r}}$, meridional $B_{\rho}$, and azimuthal $B_{\eta}$ components, measured along the corresponding axes of the local stellar coordinate system. In the observer reference frame $x y z$, the $x$ axis is pointing to the right, $y$-up, and $z$-towards the observer. Orientation of the stellar rotational axis is specified by the tilt angle of the rotation axis $i\left(0^{\circ} \leq i \leq 180^{\circ}\right)$ and the azimuth angle $\Theta\left(0^{\circ} \leq \Theta \leq 360^{\circ}\right)$, which determines orientation of the projection $C P^{\prime}$ of the rotation axis in observer $x y$ plane. The angle $\Theta$ is measured with respect to some chosen direction (e.g. towards North celestial pole), which is assumed to coincide with the $x$ axis.

Calculation of the field components in the coordinate system $x y z$ includes three transformations of the local magnetic field vector

$B^{\circ}=T_{i} T_{\eta} T_{\rho} B$ 
where $\boldsymbol{T}_{\rho}$ describes rotation around azimuthal axis of the local stellar coordinate system by the angle $90^{\circ}-\rho$,

$\boldsymbol{T}_{\rho}=\left(\begin{array}{rrr}0 & 0 & 1 \\ -\cos \rho & \sin \rho & 0 \\ \sin \rho & \cos \rho & 0\end{array}\right)$.

$\boldsymbol{T}_{\eta}$ transforms to the local coordinate system at subobserver latitude and the same longitude. This is done by the clockwise rotation around the stellar rotational axis by longitude angle $\eta$ and phase angle $\varphi$,

$\boldsymbol{T}_{\eta}=\left(\begin{array}{rrr}\cos (\eta+\varphi) & -\sin (\eta+\varphi) & 0 \\ \sin (\eta+\varphi) & \cos (\eta+\varphi) & 0 \\ 0 & 0 & 1\end{array}\right)$.

$\boldsymbol{T}_{i}$ transformation matrix corresponds to the rotation around observer $x$ axis by the inclination angle $i$ :

$\boldsymbol{T}_{i}=\left(\begin{array}{rrr}1 & 0 & 0 \\ 0 & \cos i & \sin i \\ 0 & -\sin i & \cos i\end{array}\right)$.

Finally the magnetic field vector is transformed from a Cartesian to a spherical coordinate system:

$$
\begin{aligned}
B & =\sqrt{\left(B_{x}^{\mathrm{o}}\right)^{2}+\left(B_{y}^{\mathrm{o}}\right)^{2}+\left(B_{z}^{\mathrm{o}}\right)^{2}}, \\
\chi & =\arccos \left(B_{z}^{\mathrm{o}} / B\right), \phi=\arctan \left(B_{y}^{\mathrm{o}} / B_{x}^{\mathrm{o}}\right) .
\end{aligned}
$$

On the next stage of disk integration local Stokes vectors are evaluated on an adaptive wavelength grid as described in Sect. 2.1. Local Stokes profiles are linearly interpolated onto a fine equidistant grid and convolved with the Gaussian instrumental profile using the Fast Fourier Transform (FFT). The use of the FFT algorithm allows the extra CPU time due to multiple convolutions to be kept below 3-4\% for our typical MDI problem.

The continuum flux is assumed to be constant over the whole rotational period and is determined by the disk integration in just one phase, without taking into account the presence of magnetic field or abundance spots. This assumption holds for early-type magnetic stars, but is not valid for magnetic white dwarfs, which are known to have polarized continuum radiation (Kemp 1970).

In all calculations described in our paper angle $\Theta$ was assumed to be equal to $90^{\circ}$. For the purpose of numerical experiments this can be done without loss of generality, since rotation in the observer $x y$ plane by any angle $\alpha$ modifies local or disk-integrated Stokes $Q$ and $U$ profiles by the following linear transformations (Landi Degl'Innocenti 1976):

$Q^{\prime}=Q \cos 2 \alpha+U \sin 2 \alpha$,

$U^{\prime}=-Q \sin 2 \alpha+U \cos 2 \alpha$,

while Stokes $I$ and $V$ parameters remain unchanged.

Figure 7 shows a conservative estimate of disk integration errors expected with INVERS10. For surface grids divided into 383, 695, and 1421 zones we calculated Stokes vectors for 10 rotational phases, 3 values of rotational velocity and an $8 \mathrm{kG}$ dipolar field. All other parameters were
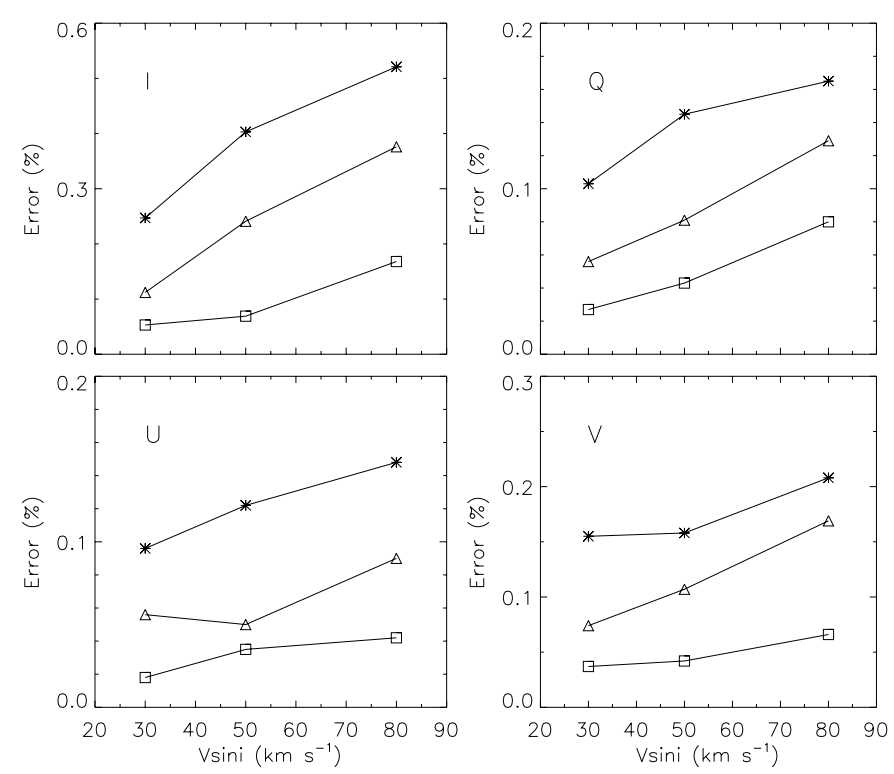

Fig. 7. Maximum disk integration errors (given in units of percent of continuum flux $I_{\mathrm{c}}$ ) of Stokes $I, Q, U$, and $V$ profiles for 3 rotational velocities and surface grids divided into 383 (asterisks), 695 (triangles), and 1421 (squares) zones. Reference Stokes profiles were calculated with spatial grid containing 3909 surface zones.

the same as in the ideal test of Sect. 3. Stokes profiles were not convolved with the instrumental profile. The results for each grid and $v \sin i$ were compared with the reference spectra, calculated on a dense spatial grid containing 3909 surface zones. Figure 7 confirms that in the case of $v \sin i=30 \mathrm{~km} \mathrm{~s}^{-1}$ the 695 -element spatial grid used throughout our calculations gives disk integration errors $\leq 0.1 \%$ for all Stokes parameters. Denser spatial grids are necessary only for the modelling of rapid rotators $(v \sin i \gtrsim$ $50 \mathrm{~km} \mathrm{~s}^{-1}$ ) observed with high $S / N$ and high spectral resolution. Apparently the number of surface elements required by INVERS10 for accurate disk integration of Stokes parameters is similar to the results obtained with far more elaborate adaptive spatial grids used by Fensl (1995).

Recently Wade et al. (2001) compared disk-integrated Stokes parameters calculated by INVERS10 with Stokes vectors generated by two other independent magnetic spectrum synthesis codes. This study showed, that once agreement on stellar parameters, model atmosphere and coordinate systems is reached, profiles calculated with three codes agree to within $0.1 \%$ of continuum flux for Stokes $I$ and to within $0.05 \%$ for Stokes $V, Q$, and $U$ profiles.

\subsection{Regularization in $\mathrm{MDI}$}

In reality rotational variability of spectral line profiles by itself does not define a unique solution of the inverse problem. Noise in the observational data, gaps in phase coverage, and intrinsic limitations of DI inversion allow an infinite number of solutions, which exhibit large fluctuations over small distances but fit observations within the error 
limits. To find a unique solution the inverse problem must be regularized. This means that a solution will be sought only within the restricted set of the surface distributions selected following some a priori criteria. Mathematically the quantity, which DI code iteratively minimizes, is represented by the sum of the measure of misfit between model and observations and an additional regularization functional (see Eq. (1)). The latter is a penalty function describing how well current solution satisfies selected criteria.

Two main forms of regularizing functional are usually employed in Doppler Imaging. Goncharsky et al. (1982) constrained the solution of the inverse problem formulated as an integral equation with a Tikhonov regularization functional. It has a general form:

$$
\Lambda R=\Lambda_{1} \sum_{i} P_{i}^{2}+\Lambda_{2} \sum_{i}\left\|\nabla P_{i}\right\|^{2}
$$

where index $i$ runs over all surface elements. In most DI applications only the part of the functional containing the gradient of the surface distribution is retained $\left(\Lambda_{1}=0\right)$. This regularization method is also implemented in INVERS10.

In their inversion code Vogt et al. (1987) adapted an alternative regularization based on Maximum Entropy Method (MEM) developed by Skilling \& Bryan (1984). The method maximizes configuration entropy:

$$
S=-\sum_{i} P_{i} \log \left(P_{i}\right)
$$

which is defined for a positive dimensionless quantity $P$.

Constraints imposed on the solutions by the Tikhonov regularization method and MEM are, in fact, different. According to Piskunov et al. (1990) "Maximizing the information entropy means that minimum correlation between different points at the stellar surface should be expected, while the use of Tikhonov regularization functional leads to the smoothest possible solution". However, despite earlier claims by Vogt et al. (1987) that only MEM produces unbiased maps and better recovers latitude information, Rice et al. (1989) and Piskunov et al. (1990) found that given high $S / N$ spectroscopic data with adequate phase coverage both regularization methods yield very similar surface distributions. In this case other limitations of DI, such as unrecognized blends or lack of information about subequatorial latitudes, play a far more prominent role than the choice of regularization functional.

In this study we again address the problem of regularization in the context of the recovery of stellar vector magnetic fields. The questions that ought to be answered include i) which regularization method is preferable for the recovery of vector components of the magnetic field, ii) which regularization scheme is appropriate for the global magnetic geometries of early-type magnetic stars and magnetic white dwarfs, and iii) does any regularization method allow successful MDI with restricted spectropolarimetric datasets (only Stokes $I$ and $V$ spectra).
One special form of regularization actively used to study magnetic fields in hot stars is parametrization. In this case the field distribution is represented by a known function or a superposition of functions (e.g. low-order spherical harmonics) and the minimization is reduced to a search for an optimal set of parameters.

For the first time such approach was successfully applied to the MDI based on spectropolarimetric data by Piskunov et al. (1985) and further developed by Landstreet (1988). Recently Vasilchenko et al. (1996) combined it with the conventional Doppler reconstruction of abundance maps.

However, such parameter optimization is necessarily biased towards a far narrower class of solutions than the regularized MDI problem, and thus generally fails to reveal the structure of the magnetic field permeating stellar atmospheres. Even if the best-fit multipolar field is found for a particular object, possible deviations from this model cannot be readily quantified in terms of local photospheric magnetic parameters, nor can the model itself easily be proven to be unique. Besides, a consensus about the form of multipolar parametrization is yet to be reached (superposition of aligned dipole, linear quadrupole, and octupole used by Landstreet \& Mathys (2000); arbitrary shifted dipole of Khokhlova et al. (1997); and a combination of dipole and non-axisymmetric quadrupole from the study by Bagnulo et al. (2000) do not exhaust all forms of multipolar expansion suggested in recent publications on early-type magnetic stars.)

Recent Zeeman-Doppler Imaging results presented by Donati et al. (Donati 1999; Donati et al. 1999) confirmed the long-held belief that late-type active stars possess complex magnetic fields without any stable global structures. The nature of such fields allows relatively straightforward extension of the conventional regularization methods to the imaging of vector magnetic fields. Indeed, the Tikhonov regularization functional is defined for positive as well as negative values of the surface distribution $P$. However, the entropy expression (56) holds only for strictly positive, non-zero quantities. Therefore for the purpose of the recovery of vector field, which can take both negative, positive, or zero values, entropy definition must be reformulated. Brown et al. (1991) used an entropy functional

$S=-\sum_{i}\left(\left|P_{i}\right|+\alpha\right)\left[\log \frac{\left|P_{i}\right|+\alpha}{\left|m_{i}\right|+\alpha}-1\right]$

where $m_{i}$ is the default value assigned to the surface pixel $i$ and $\alpha$ is small positive number, which ensures that entropy is defined for zero field values.

Hussain et al. (2000) discuss two other forms of entropy functionals implemented in their independent magnetic DI codes. Firstly,

$S=-\sum_{i}\left[f_{i} \log \frac{f_{i}}{m_{i}}+\left(1-f_{i}\right) \log \frac{1-f_{i}}{1-m_{i}}\right]$ 

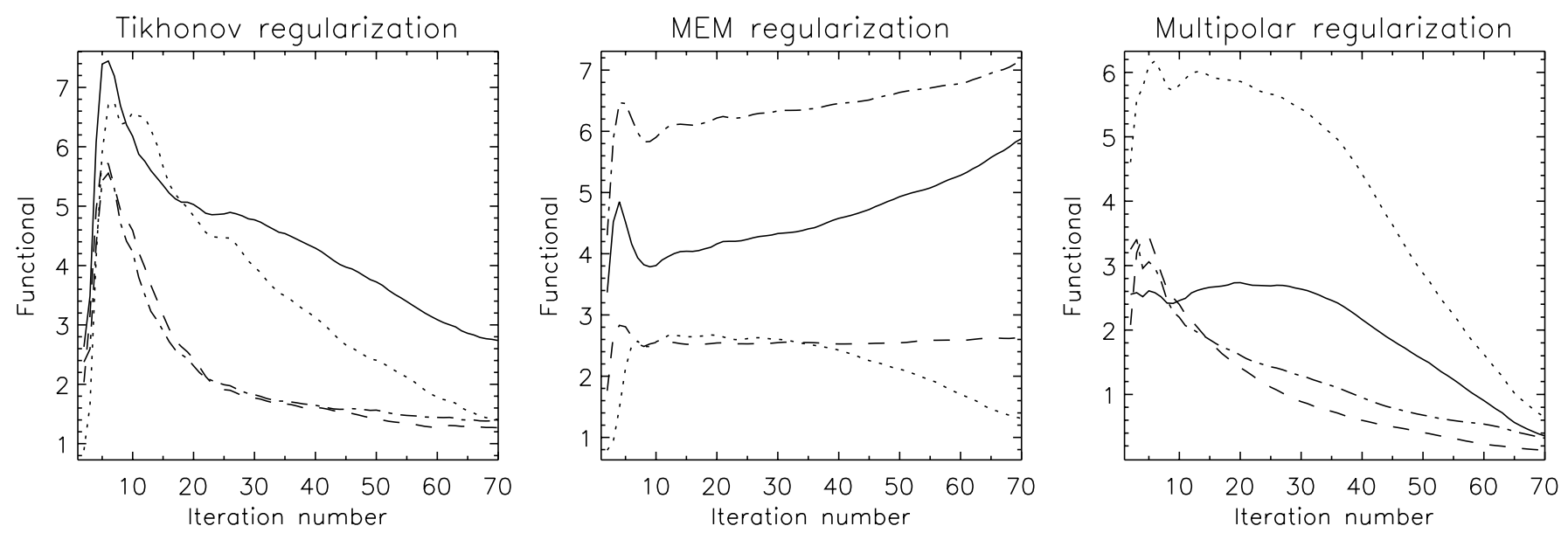

Fig. 8. Comparison of Tikhonov, maximum entropy, and multipolar regularization functionals (given in arbitrary units) for ideal MDI reconstruction of a dipolar field. The solid curve shows functionals computed for a radial field map, while dotted, dashed, and dash-dotted curves correspond to the meridional component, azimuthal component, and field modulus, respectively.

where $f_{i}$ is a spot filling factor

$f_{i}=\frac{P_{i}+P_{\max }}{2 P_{\max }}$

with $P_{\max }$ being equipartition value adopted for the star (Hussain et al. used $P_{\max }=5 \mathrm{kG}$ ). Alternatively one can use definition

$$
\begin{aligned}
& S=\sum_{i}\left[\phi_{i}-m_{i}-P_{i} \log \frac{\phi_{i}+P_{i}}{2 m_{i}}\right], \\
& \phi_{i}=\sqrt{P_{i}+4 m_{i}^{2}} .
\end{aligned}
$$

Hussain et al. report that application of two different forms of MEM regularization to the recovery of surface magnetic maps of the young active star AB Dor resulted in slightly different contrasts of the magnetic distribution. In particular the code with entropy defined following expression (60) tends to produce images with stronger features covering smaller area, while application of formula (58) produces images with weaker features spread out over larger areas.

Comparison of entropy functional (57), suggested by Brown et al. (1991), with definitions (58) or (60) shows that in principle one can expect to find even larger discrepancies in the maps regularized by these functions. This conclusion comes from the fact that in expression (57) positive and negative field values of the same magnitude are indistinguishable, while functionals (58) and (60) are non-symmetric in respect to the field sign. Thus a certain influence of the form of adopted entropy functional on the final magnetic maps should not be forgotten when discussing results of MDI with MEM-based codes. Apart from this uncertainty, maximum entropy regularization as well as the Tikhonov method seems to be perfectly acceptable for the imaging of non-global stellar magnetic fields. Numerical experiments conducted by Brown et al. (1991) and Donati \& Brown (1997) (who used a MEM DI code) and our results based on the Tikhonov regularization method (will be presented in a forthcoming paper) confirm the ability of both MDI codes to recover positions and sizes of the magnetic spots. Orientation of the field is recovered with good precision when all four Stokes parameters are used in the inversion, but strong cross-talk between radial and meridional field components is observed in the case of imaging with circular polarization data only.

Surprisingly enough, recovery of the strong globallyorganized fields in chemically peculiar stars and magnetic white dwarfs appeared to be substantially more problematic than imaging weak magnetic spots on late-type active stars. Brown et al. (1991) and later Donati et al. (1994) failed to recover magnetic dipoles with polar strengths typical for a magnetic Ap star and a white dwarf, respectively. Both studies used maximum entropy regularization and extracted magnetic maps from the variability of synthetic Stokes $I$ and $V$ parameters. Recently Donati (2001) reported the failure of a similar MDI code to recover the structure of the magnetic dipole even from rotational modulation of all four Stokes parameters. However, in our study we found that the MDI code INVERS10 is capable of accurate reconstruction of the dipole and quadrupole fields using four Stokes parameters and the Tikhonov regularization technique. At the same time attempts to apply the same scheme to $I V$ Doppler reconstruction of the global fields were not successful.

In order to clarify the difference between the two regularization schemes (MEM and Tikhonov) and to understand their failure in the case of $I V$ imaging we monitored variations of the regularization functionals during ideal magnetic inversion (Sect. 3). Observational material used in this test consisted of 20 phases of Stokes vectors with very high $S / N$, distributed uniformly over the rotational period. Due to low noise and dense phase coverage of the synthetic spectropolarimetric data we expect the reconstruction to be governed mostly by the information 
contained in line profiles, while regularization will play a relatively minor role.

At every iteration values of different regularization functionals were determined for magnetic distributions and compared with each other. For the MEM functional the negative (since our code minimizes the total discrepancy function) of Hussain et al. (2000) definition (58) was adopted. Results of the comparison are shown in Fig. 8. We found that in the case of Tikhonov regularization functionals of all three vector components and modulus of the magnetic field decrease rapidly in the first 1520 iterations and then continue to decrease albeit not as steeply. The situation with the maximum entropy functionals is markedly different. Only the functional of the meridional component shows a tendency to decrease as it should, for other components and for the field modulus the maximum entropy requirement is not improving in the course of inversion: configuration entropy of the corresponding magnetic maps either decreases (field modulus and radial component) or stays approximately constant (azimuthal component). Such behaviour is to be expected, since MEM tries to minimize informational content of the surface maps by prohibiting large-scale deviations from the average value of a parameter of interest. The structure of the global stellar magnetic fields is just the opposite of what MEM-based code seeks to find. The global magnetic map consists of smooth large-scale deviations from the average rather than isolated spots on the homogeneous background. In other words, a low-order multipolar field does not have minimal informational content among all solutions permitted by the observational data. Thus, the maximum entropy method is not the best choice for imaging global magnetic fields. The Tikhonov regularization scheme is more appropriate for such DI problems. However, this form of penalty function also has its own limitations. It works well only when the role of regularization is small, like in the imaging with complete Stokes vector data. Still, the basic assumption of the Tikhonov method - the selection of the smoothest surface map - is not valid for multipolar fields. This is illustrated in Fig. 9, where we display meridional gradients computed for the vector components of a magnetic dipole and quadrupole. Even for the simplest dipolar field intrinsic variations of the gradient are relatively high. According to Fig. 9 these variations quickly increase in amplitude with the departures from dipolar magnetic geometry. Therefore, minimization of the gradient will not lead to the correct solution as confirmed by our tests of $I V$ magnetic imaging.

Nevertheless, interpretation of high-resolution spectropolarimetric observations in Stokes $I$ and $V$ parameters can be achieved by means of magnetic DI. This can be done by introducing a new regularization method that we call multipolar regularization. In contrast with the MEM and Tikhonov methods, which adopt very general informational and smoothness criteria, our regularization scheme uses specific empirical knowledge that a superposition of low-order multipoles most easily accounts for the main properties of the observational data in the presence of

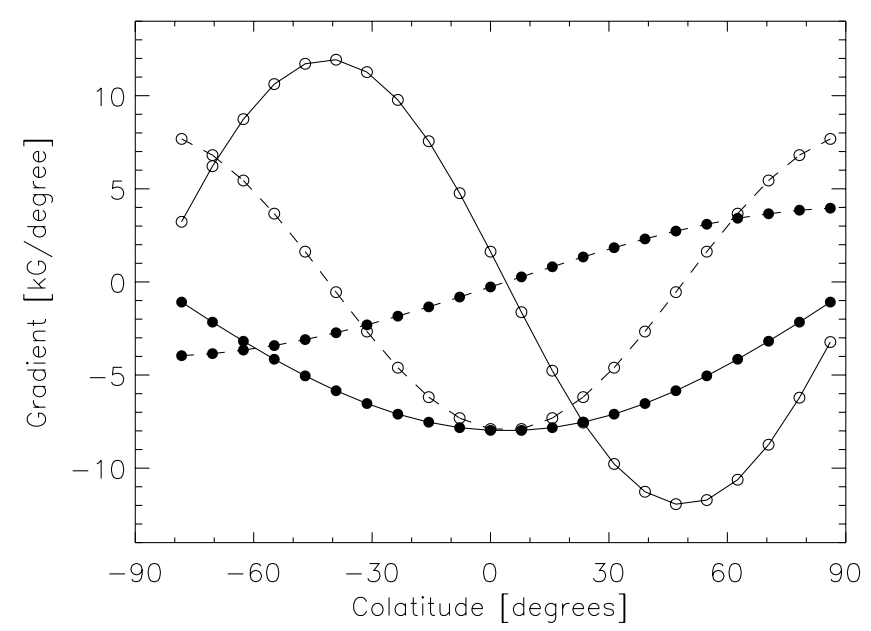

Fig. 9. Colatitude dependence of the meridional gradient computed for radial (solid curve) and meridional (dashed curve) components of an $8 \mathrm{kG}$ dipole (filled circles) and an $8 \mathrm{kG}$ quadrupole (open circles) fields.

global fields. Therefore, it is reasonable to suggest that one should search for a surface distribution, which is close to some multipolar field. To select such a magnetic map among all possible images we add a new regularizing functional to the total discrepancy function (1)

$\Psi=\chi^{2}(Z, \boldsymbol{B})+\Lambda_{1} R_{1}(Z)+\Lambda_{2} R_{2}(\boldsymbol{B})+\Lambda_{3} R_{3}(\boldsymbol{B})$,

where $Z$ and $\boldsymbol{B}$ are surface abundance and magnetic distributions respectively, $R_{1}$ is conventional (Tikhonov) regularization functional necessary for proper recovery of the abundance map, $R_{2}$ is a Tikhonov functional for the magnetic field, $R_{3}$ is the multipolar regularization function, and $\Lambda$ 's are corresponding regularization parameters. (We found it practical not to abandon Tikhonov regularization completely. It helps to smooth the field distribution on the first iterations when $\Lambda_{2} R_{2}(\boldsymbol{B}) \approx \Lambda_{3} R_{3}(\boldsymbol{B})$; after that the multipolar term dominates and the contribution of $\Lambda_{2} R_{2}(\boldsymbol{B})$ is negligible.) During every iteration we evaluate the functional $R_{3}$ as

$R_{3}=\sum_{i}\left(\boldsymbol{B}_{i}-\boldsymbol{B}_{i}^{\text {model }}\right)^{2}$

where $\boldsymbol{B}$ is the current magnetic field, $\boldsymbol{B}^{\text {model }}$ is the bestfit model configuration derived for the current magnetic distribution, and summation is carried out over all surface zones.

With our modification of the regularization procedure MDI code has a tendency towards selecting a magnetic distribution close to the specified functional form, rather than just searching for the smoothest surface distribution. This new regularization does not depend on the method of the search and parametrization of the optimal model field and therefore the regularization procedure can be easily modified to use any prior knowledge about the type of magnetic field geometry expected for a particular class of objects. For INVERS10 we adopted a low-order multipole 
expansion, which is known to give good results for magnetic chemically peculiar stars and white dwarfs. A multipolar model is determined by minimizing the functional similar to $(62)$

$\Re=\sum_{i} \omega_{i}\left(\boldsymbol{B}_{i}-\boldsymbol{B}_{i}^{\text {model }}\right)^{2}$.

In all stellar DI applications sub-equatorial part of the reconstructed map is the least reliable, therefore we use additional weights $\omega$ to restrict the influence of these regions on the derived model field. In the current implementation of multipolar regularization in INVERS10 only latitude dependence is included in weight function:

$\omega=\left\{\begin{array}{ll}\sin (\rho+i) & , \text { for } \rho \geq-i \\ 0 & , \text { for } \rho<-i\end{array}\right.$,

where $\rho$ is latitude on the stellar surface and $i$-inclination angle of the stellar rotational axis. This form of weight function closely follows latitude variation of the average discrepancy between the true and reconstructed magnetic maps. In practical applications information about relative $S / N$ of observational data and its phase distribution may also be taken into account in $\omega$.

The method of solving the weighted least-squares problem (63) is detailed in Appendix A. It is based on the solution of the system of linear equations, which yields a unique set of coefficients of the multipolar expansion into real spherical harmonics. This parametrization of global magnetic fields is in fact equivalent to the model proposed by Bagnulo et al. (1996) (combination of dipole and general non-linear quadrupole, octupole, etc.). In this scheme multipolar components are not aligned, and therefore non-axisymmetric field distributions are possible. The current implementation of the multipolar regularization in INVERS10 includes harmonics up to second order.

Multipolar regularization gives several important advantages over direct minimization of the multipolar field parameters. First it is possible to control the stability of the MDI problem in the case of insufficient data (e.g. when linear polarization data is of low quality or absent). Second, although with this regularization the code tends to select multipolar fields, the solution will not be restricted to such a model if it is inconsistent with the observations. Third, the new regularization conveniently provides an estimate of the multipolar field geometry at every iteration and allows better results for the reconstruction of magnetic field in the low-latitude surface regions to be achieved.

Multipolar regularization is also very useful for the interpretation of MDI maps obtained with INVERS10 and for the comparison with the results obtained by other methods.

Figure 8 confirms that the multipolar functional computed for all vector components and modulus of the dipolar field steadily decreases through the whole inversion procedure. Note, that multipolar regularization was switched off during solving this problem and was not in any way influencing the search for a solution.

\subsection{Minimization}

We started by using the conjugate gradient method (e.g. Bevington \& Robinson 1992) as the minimization algorithm. The main advantage of the method is the ability to handle a large number of variables with modest memory requirements. The disadvantage is that the method minimizes the scalar function and therefore with a large number of observables the changes of the minimized function close to minimum are strongly influenced by rounding errors. In addition, although gradient search is ideal for approaching the minimum from far away, it converges slowly near the minimum. With the conjugate gradient method INVERS10 needs $75-150$ iterations to achieve convergence for a typical MDI problem.

The more advanced Marquardt-Levenberg (ML) minimization algorithm (Bevington \& Robinson 1992) combines the best features of the gradient search with the method of linearizing the fitting function, which ensures rapid convergence close to the minimum. In the ML method one usually finds corrections for unknown parameters $x$ by solving the system of linear equations

$\alpha^{\prime} \delta x=\beta$

for each iteration. Vector $\boldsymbol{\beta}$ is proportional to the first derivatives of the total discrepancy $\Psi$ :

$\beta_{k} \equiv-\frac{1}{2} \frac{\partial \Psi}{\partial x_{k}}=-\frac{1}{2} \frac{\partial \chi^{2}}{\partial x_{k}}-\frac{\Lambda}{2} \frac{\partial R}{\partial x_{k}}$,

while $\alpha^{\prime}$ is the curvature matrix with a modified main diagonal

$\alpha_{i k}^{\prime}=\alpha_{i k}\left(1+\tau \delta_{i k}\right)$,

$\alpha_{i k} \equiv \frac{1}{2} \frac{\partial^{2} \Psi}{\partial x_{i} \partial x_{k}}=\frac{1}{2} \frac{\partial^{2} \chi^{2}}{\partial x_{i} \partial x_{k}}+\frac{\Lambda}{2} \frac{\partial^{2} R}{\partial x_{i} \partial x_{k}}$.

Near the minimum, where computed Stokes spectra $\boldsymbol{I}_{\phi \lambda}^{\mathrm{com}}$ are sufficiently close to the observations $\boldsymbol{I}_{\phi \lambda}^{\text {obs }}$, second derivatives of the $\chi^{2}$ can be approximated with

$$
\begin{aligned}
& \frac{1}{2} \frac{\partial^{2} \chi^{2}}{\partial x_{i} \partial x_{k}}=\frac{1}{2} \frac{\partial^{2}}{\partial x_{i} \partial x_{k}} \sum_{\phi \lambda} \frac{1}{\sigma_{\phi \lambda}^{2}}\left[\boldsymbol{I}_{\phi \lambda}^{\mathrm{obs}}-\boldsymbol{I}_{\phi \lambda}^{\mathrm{com}}\right]^{2} \\
& \quad=\sum_{\phi \lambda} \frac{1}{\sigma_{\phi \lambda}^{2}}\left[\frac{\partial \boldsymbol{I}_{\phi \lambda}^{\mathrm{com}}}{\partial x_{i}} \frac{\partial \boldsymbol{I}_{\phi \lambda}^{\mathrm{com}}}{\partial x_{k}}-\left(\boldsymbol{I}_{\phi \lambda}^{\mathrm{obs}}-\boldsymbol{I}_{\phi \lambda}^{\mathrm{com}}\right) \frac{\partial^{2} \boldsymbol{I}_{\phi \lambda}^{\mathrm{com}}}{\partial x_{i} \partial x_{k}}\right] \\
& \quad \simeq \sum_{\phi \lambda} \frac{1}{\sigma_{\phi \lambda}^{2}} \frac{\partial \boldsymbol{I}_{\phi \lambda}^{\mathrm{com}}}{\partial x_{i}} \frac{\partial \boldsymbol{I}_{\phi \lambda}^{\mathrm{com}}}{\partial x_{k}}
\end{aligned}
$$

while second and first derivatives of the regularization functions $R$ are evaluated using explicit analytical expressions.

The scalar parameter $\tau$ controls interpolation of the ML algorithm between the gradient search and secondorder expansion of the fitting function. In the modified ML method that we have implemented in the latest version of INVERS10 the adjustment of $\tau$ is made several times within each iteration by finding the value of $\tau$ which minimizes 
the total discrepancy $\Psi$ for a given curvature matrix $\alpha$ and right-hand side vector $\beta$. When the optimal $\tau$ is found and $\Psi$ can no longer be decreased, the minimization algorithm recomputes the curvature matrix and $\beta$ and repeats adjustment of the $\tau$ parameter. We found this minimization method to be much more efficient and stable, ensuring convergence in typically 10-15 iterations. However, calculation of the curvature matrix with expression (69) requires operating with very large matrices which can easily exceed available memory resources even for modest MDI problems.

\subsection{Parallel execution}

INVERS10 is designed for parallel execution. We used the Message Passing Interface (MPI) for organizing the interaction between processes. The initial concept is based on the fact that during each iteration spectral synthesis for each rotational phase or surface element can be performed independently. The original single-program-multiple-data implementation of INVERS10 begins with reading in the observational data and the starting model and initialization of arrays that can be pre-computed. These data are distributed between all processes. After that, each process takes approximately an equal number of phases and computes spectral synthesis and derivatives of the local line profiles for the current distribution of the magnetic field and abundance. It is useful to have the number of processes equal to the number of rotational phases that needs to be synthesized. The main process collects computed spectra, corrects the maps and distributes them to all processes for the next iteration.

Although the code based on this concept performs satisfactory and scales almost linearly with the number of CPUs, it has two important limitations:

- no performance improvement is possible after the number of CPUs has reached the number of observed phases;

- load balance is difficult to achieve. Each iteration takes as long as the slowest process which does approximately as much work (phases) as the other faster processes.

An alternative MPI-based algorithm for parallel execution has been implemented in the latest version of INVERS10. (In this improved version of the MDI code we have also implemented the modified Marquardt-Levenberg minimization algorithm described in the previous section). This new parallel scheme consists of the following steps:

$1^{\circ}$ Main process reads input data and initializes subordinate processes (radiative transfer solvers).

$2^{\circ}$ Main process starts minimization of the total discrepancy function $\Psi$.

$3^{\circ}$ Local Stokes $I, Q, U$ and $V$ spectra and their derivatives are computed by multiple radiative transfer solvers. $4^{\circ}$ Main process performs disk integration and continues minimization.

$5^{\circ}$ When the minimum is achieved, the main process signals to subordinate processes to exit.

During step $3^{\circ}$, when disk integration is done, the main process:

I Takes the next surface element and sends its parameters for all rotational phases to the first available radiative transfer solver. This step is repeated, without waiting, until there is no free solver available.

II Waits for any solver to complete the calculations.

III When this happens, the main process retrieves local Stokes profiles and their derivatives and updates respective disk integrals.

IV If there are unprocessed surface elements left, passes the next one to this radiative transfer solver and goes back to II.

Meanwhile each subordinate process (radiative transfer solver):

I Receives a surface element (local abundances, magnetic field vector and orientations relative to the lineof-sight) from the main process.

II Constructs the total opacity matrix $\mathcal{K}$ and solves the radiative transfer equation for four Stokes parameters and numerically evaluates derivatives with respect to local parameters. Calculations are repeated for all rotational phases and are carried out on an adaptive wavelength grid which ensures accurate linear interpolation between different wavelengths samples. Next, polarization spectra are interpolated onto a fine equispaced wavelength grid and convolved with the instrumental profile using a FFT (see Sect. 2.6).

III Signals the completion to the main process.

IV Returns the results and waits for the next surface element.

Since the time spent on computing Stokes parameters for each surface element in each rotational phase dominates the magnetic DI procedure the advantages of such an implementation are obvious:

- The data traffic is negligible compared to the computing load, therefore the total time for a single iteration is reduced proportionally to the number of available processors assigned to solve the radiative transfer. This will scale linearly until the number of processors matches the number of surface elements (700-2000).

- The CPU load is automatically balanced: a faster radiative transfer solver process does calculations for more surface elements.

- Further speed increase can be achieved by taking advantage of the fact that some computationally expensive functions (e.g. Voigt and Faraday-Voigt) do not depend on the rotational phase (Zeeman splitting is proportional to the field strength and is not influenced by field orientation) so they have to be evaluated only at one phase for each surface element. 

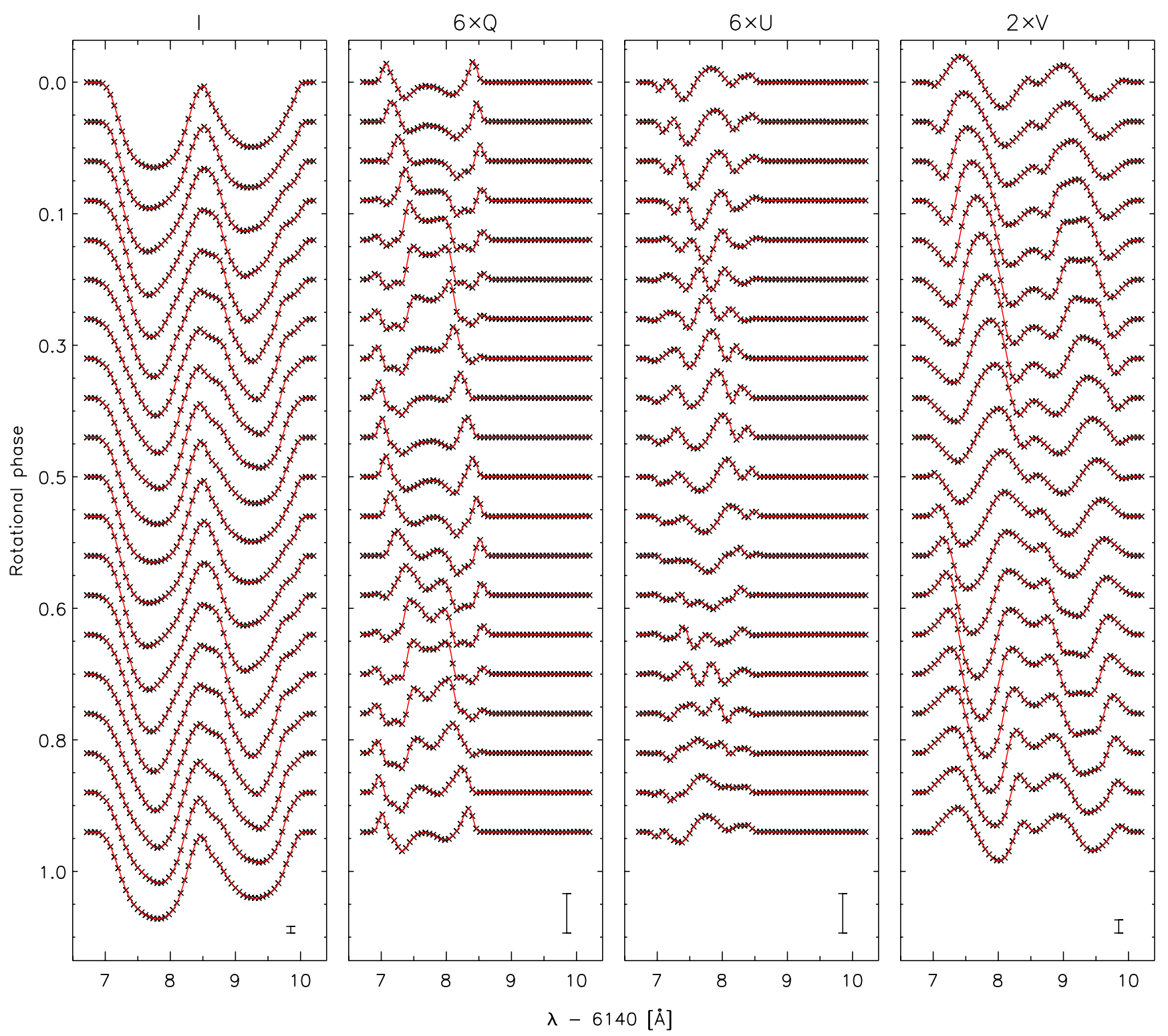

Fig. 10. Synthetic Stokes $I, Q, U$ and $V$ profiles of Fe II 6147.74 and $6149.26 \AA$ spectral lines for the dipolar magnetic distribution (Fig. 11) adopted in the ideal MDI reconstruction test. Simulated observational data is shown by symbols, while solid lines represent the final fit of MDI code. Profiles of consecutive phases are shifted in vertical direction. Note the different scale used for Stokes $I$ profiles, circular and linear polarization. The bar at the lower right of each Stokes parameter plot corresponds to $1 \%$ of the continuum level of Stokes $I$.

- Local memory per process has (modest) dimensions of the observed data set.

- Implementation of the radiative transfer solvers as a separate program allows switching between different numerical algorithms without modification of the main component of INVERS10.

\section{Ideal MDI reconstruction}

Numerical experiments in which an MDI code is applied to recover the surface distribution from simulated observational datasets is the most direct and reliable method to test the performance of a Doppler Imaging code. Due to the enhanced complexity of the magnetic
DI reconstruction in comparison with abundance or temperature mapping, any MDI code should undergo extensive and rigorous tests in order for its intrinsic limitations and sensitivity to observational data to be assessed properly. We plan to describe these extensive numerical experiments with INVERS10 in the forthcoming paper, while here we limit ourselves to the discussion of MDI reconstruction from a noiseless four Stokes parameters dataset with a good coverage of a stellar rotational period. Such an ideal MDI reconstruction will serve as a benchmark for studying what in principle can be achieved with the application of magnetic DI method to the analysis of global magnetic fields of CP stars and magnetic white dwarfs. Despite the fact that far more complex magnetic fields of 

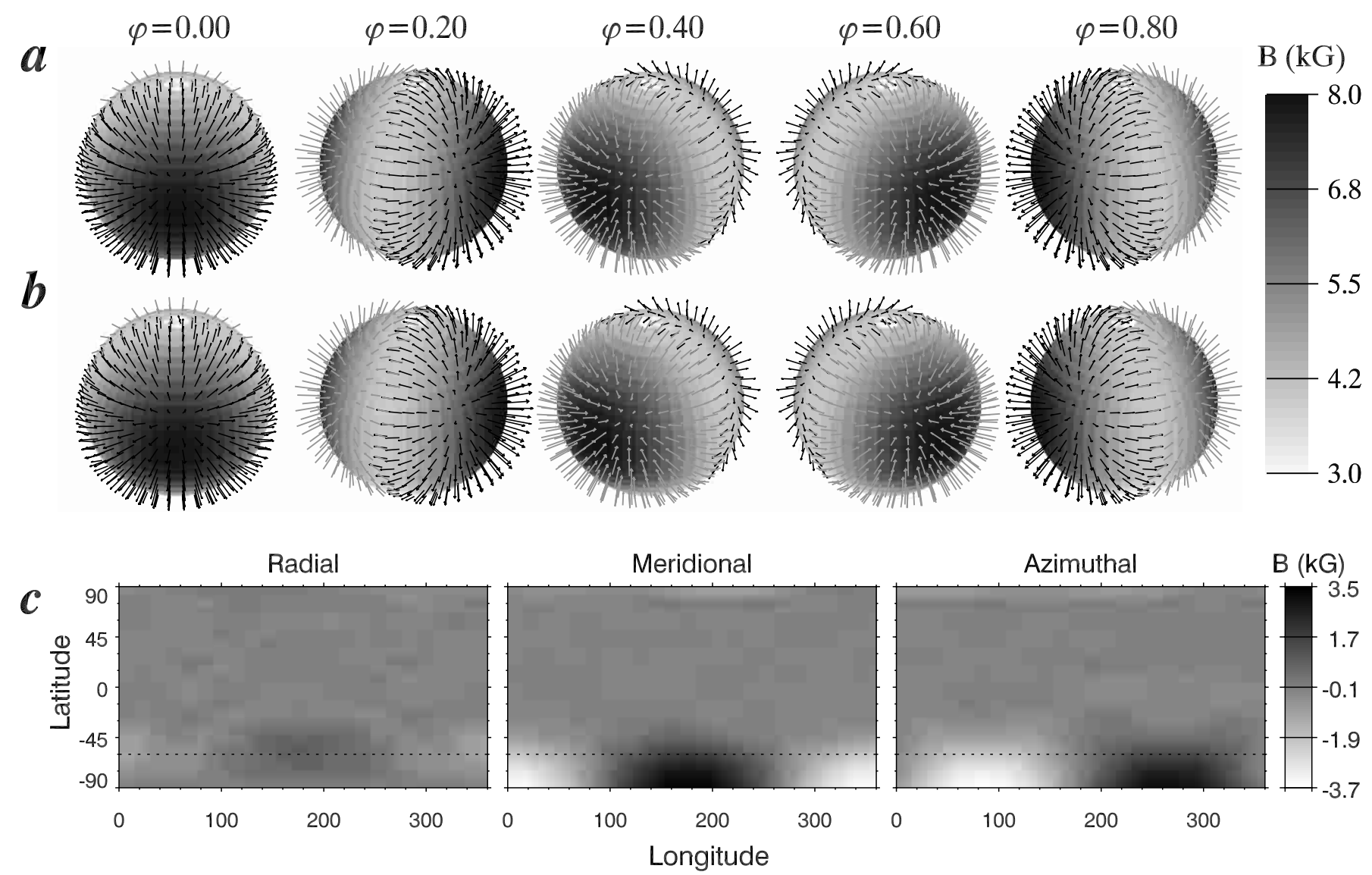

Fig. 11. Comparison between the true dipolar magnetic distribution a) and the vector magnetic map b) recovered in an ideal magnetic DI reconstruction test. Black arrows show field vectors pointing outside the stellar surface, while grey arrows correspond to the vectors pointing inwards. Arrow length is proportional to the field strength. Underlying grey shades of images a) and b) visualize the distribution of the magnetic field modulus. Panel c) shows rectangular projections of the difference between radial, meridional and azimuthal vector components of the true and reconstructed magnetic images. The dotted line corresponds to the lowest visible latitude for the adopted inclination angle $i=60^{\circ}$.

late-type stars are now routinely reconstructed using only Stokes $I$ and $V$ profiles (e.g. Hussain et al. 2000), the very possibility of non-parameterized MDI of global magnetic fields of CP stars, even using four Stokes profiles datasets, has been questioned by some authors (e.g. Donati 2001). Therefore, it is important to check if one can recover a global magnetic distribution without any a priori assumptions about the magnetic field geometry. In addition, the MDI reconstruction described in this section is used for the analysis of the behaviour of the regularization functionals (see Sect. 2.7) as well as for the study of variance properties of a reconstructed image (see Sect. 4).

We calculated artificial four Stokes parameter observations using a solar metallicity ATLAS9 (Kurucz 1993) model atmosphere with $T_{\text {eff }}=9000 \mathrm{~K}$ and $\log g=4.0$. The model star was assumed to rotate with $v \sin i=30 \mathrm{~km} \mathrm{~s}^{-1}$ and inclined by $i=60^{\circ}$ with respect to the line-of-sight. For the magnetic geometry we used a dipolar field with $B_{\mathrm{d}}=8 \mathrm{kG}$ and magnetic axis inclined by $90^{\circ}$ with respect to the rotational axis, so that the dipole lies in the stellar equatorial plane and its positive pole crosses the plane containing the line-of-sight and stellar rotational axis at phase zero. The synthetic Stokes parameters were computed for 20 equidistant rotational phases and broadened with a Gaussian instrumental profile corresponding to a resolution of $\lambda / \Delta \lambda=100000$. Stokes profiles were synthesized for two Fe II lines at 6147.74 and $6149.29 \AA$ assuming an iron abundance $\varepsilon(\mathrm{Fe}) \equiv \log \left(N_{\mathrm{Fe}} / N_{\text {tot }}\right)=$ -3.65 . Lines in this iron doublet are known to have very different Zeeman splitting patterns and hence their simultaneous analysis can benefit from extracting information about the magnetic field distribution not only from circular and linear polarization profiles, but from Stokes $I$ spectra as well. The initial guess consisted of an homogeneous iron distribution with $\varepsilon(\mathrm{Fe})=-4.0$ and zero magnetic field everywhere on the stellar surface. The magnetic field vector map and abundance images were adjusted simultaneously using Tikhonov regularization but without applying the multipolar regularization scheme introduced in Sect. 2.7.

The final fit to the simulated observational dataset is illustrated in Fig. 10. Figures 11a and b compare reconstructed magnetic distributions with the true magnetic image, while panel c in Fig. 11 shows rectangular maps of the difference between true and reconstructed magnetic field vector components. Results of the ideal magnetic 
Doppler reconstruction confirm that there are no principle obstacles for recovering a global magnetic field once high quality observations in four Stokes parameters are used for MDI recovery. Over the significant part of the visible stellar surface INVERS10 converged on a magnetic distribution that was different from the true dipolar map by only 100-200 G. The iron abundance was also recovered very well, with the final abundance map deviating from the true $\varepsilon(\mathrm{Fe})$ by no more than 0.05 dex and without any signs of crosstalk between magnetic and abundance images. The quality of the reconstruction decreases slightly towards the stellar rotational pole and lower latitude belts, while the discrepancy between true and recovered magnetic maps reaches a few $\mathrm{kG}$ for unseen latitudes (those below $\left.-60^{\circ}\right)$. This behaviour is consistent with what one expects from any other stellar Doppler Imaging application based on the analysis of rotational line profile variability. Thus, the main conclusion of this section is that magnetic Doppler Imaging of global fields of CP stars (and white dwarfs) does not need to be carried out using such a priori assumptions about global field geometry as, for example, parametrization with low-order multipolar expansion. As soon as a high-quality observational dataset of circular and linear polarization inside line profiles is obtained, reliable imaging of global stellar magnetic fields can be performed regardless of the geometrical characteristics and level of global organization of the fields on surfaces of particular classes of magnetic stars. This opens the possibility to apply the same MDI code for consistent mapping of magnetic field geometries across the whole HR diagram.

\section{Error analysis}

In all DI studies assessment of the image variance should in principle complement numerical or visual comparison of true images and distributions, recovered in test experiments. Nevertheless, formal error analysis of reconstructed surface maps has received relatively little attention in DI simulations and practical applications. Recently Berdyugina (1998) introduced the Occamian approach for regularization of the ill-posed problem of imaging temperature surface distributions from time series of unpolarized line profiles. As the by-product, lower limits of errors in recovered temperature maps were estimated. Berdyugina claimed that possibility of such error estimates is an advantage of the Occamian method, and errors cannot be computed with other regularization schemes. This assertion is not correct, since formal error analysis can be carried out in every minimization problem, regardless of the exact form of regularization functional. In particular, variance estimates for each pixel of the recovered image can be conveniently obtained from the main diagonal of the inverse of the curvature matrix:

$\sigma_{k k}^{2}=\left(\alpha^{-1}\right)_{k k}$,

where the matrix $\alpha$ is defined by (68). Since $\Psi$ represents the total discrepancy of the regularized inverse problem, the matrix $\alpha$ is well-conditioned and its inverse exists.
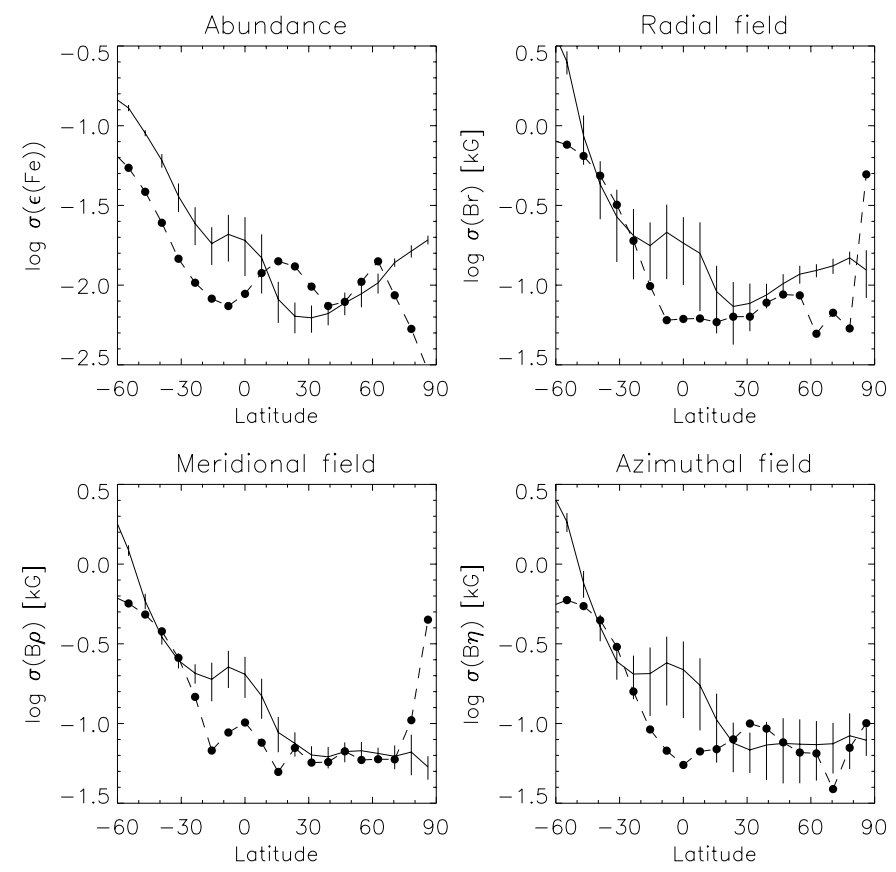

Fig. 12. Latitude dependence of the formal variance, computed for abundance and three components of the vector magnetic field. Solid lines show latitude variation of $\sigma$, vertical dashes correspond to \pm one standard deviation of the variance scatter within each latitude belt. Filled circles show the latitude trend of the mean absolute deviation between recovered and true distributions.

Thus, once first derivatives of the synthetic Stokes profiles $\boldsymbol{I}_{\phi \lambda}^{\text {comp }}$ and second derivatives of the regularization functional $R$ are evaluated, formal error estimates $\sigma$ can be computed with the expressions (70).

In Fig. 12 we compare $\sigma$, computed from the curvature matrix, with absolute deviations, estimated by direct comparison of recovered maps and true test distributions. These calculations were performed for the converged ideal test model (Sect. 3) with an homogeneous Fe distribution. Since the $\sigma$ and absolute deviation images possess mostly latitude dependence, we averaged them in the longitudinal direction and estimated the scatter within latitude belts by computing standard deviations for each latitude.

Formal and direct error estimates agree relatively well, in the sense that absolute deviations lie within a $3 \sigma$ envelope, set by the formal variance. Note that divergence of the curves for the lowest visible latitudes, where formal errors increase faster than the difference between true and recovered images, is not inconsistent. Abundance and field vectors recovered for the low-latitude surface regions strongly depend on the adopted initial surface distributions, and therefore absolute discrepancy between lowlatitude parts of the true and recovered maps will also be determined by the initial guess. Variance curves, on the other hand, reflect the true range of the solutions, which fit the data within the error limits and for a given regularization parameter $\Lambda$. Variance distributions shown in Fig. 12 are roughly consistent with the major $\sin (\rho+i)$ dependence (with the exception of the equatorial bump in $\sigma$ 
distributions, which is associated with magnetic poles). This gives a posteriori justification of the weight function adopted for the multipolar regularization (Sect. 2.7).

Error distributions published by Berdyugina (1998) are also dominated by pole-to-equator variations, but, unlike latitude dependencies of Fig. 12, their minima are located on the visible rotational pole, regardless of the stellar inclination $i$. However, from pure symmetry considerations one would expect to find the minimum of the latitude error distribution close to the subobserver latitude, which depends on the adopted inclination angle and smoothly changes from the stellar rotational equator (for $i=90^{\circ}$ both poles are visible and latitude variance distribution must be symmetrical with respect to equator) to the visible rotational pole (for $i=0^{\circ}$ ). This inconsistency suggests that lower limits of the errors, computed with an Occamian approach, do not fully resolve latitude trends of the image variance and are not very useful in DI.

\section{Conclusions}

We have presented a new code capable of reconstructing the vector distribution of the magnetic field on the surface of an Ap star together with the abundance of one chemical element. The aim of constructing such a code is more general, namely, to build a tool which (with small modifications) can be applied to study the actual field geometry in stars at different evolutionary stages and masses. The initial application is aimed at magnetic Ap stars because the observational data is available and the polarization signal is strong, but eventually systematic polarization measurements will be obtained for classical $\mathrm{T}$ Tauri stars, active close binaries, active solar-type stars etc. allowing us to develop realistic models (accretion, mass exchange, dynamo) for these systems based on derived field geometries.

Embarking on this project we realized that we have to study a number of related areas to be sure that the building blocks are reliable and ensure the performance of the whole method. The results of such studies have been presented here so that our work can be understood by other groups and the results can be reproduced.

In particular, we have compared the performance of different magnetic RT solvers and found that Feautrier and quadratic DELO are sufficiently fast and show the best results in terms of accuracy and convergence. The disk integration scheme combines fixed geometry and high accuracy even on a rather sparse grid. An ideal MDI problem appears to have a unique solution (we will return to this issue in the second paper in this series) therefore conventional regularization methods (Tikhonov and MEM) are useful to stabilize the inversion for the discrete wavelength and phase datasets. We also show that the Tikhonov form of the regularization function is more compatible with the global field geometries (low-order multipoles), while MEM can be more suitable for the solartype fields. In the case of partial polarization data (e.g. Stokes $I$ and $V$ only) the problem does not have a unique solution. Special multipolar regularization was developed for Ap stars which restricts the class of possible solutions in these situations but allows deviations from a simple combination of low-order spherical harmonics.

We have switched to the more efficient MarquardtLevenberg minimization algorithm in order to reduce the computation time and avoid problems caused by rounding errors. We also describe the parallel implementation of our code based on the MPI concept which allows us to achieve nearly linear scaling of performance with the number of CPUs.

We have also developed an error analysis procedure which is capable of giving an error bar for each surface element of the recovered maps. The comparison of the formal error estimates with the actual differences between the reconstruction and the exact solution for the test cases confirms the usefulness of this procedure.

In the coming papers we will present the results of numerical experiments, study the effects of systematic errors in stellar parameters and different regularization strategies.

\section{Appendix A: Least-squares harmonic decomposition of the vector field}

Here we address a general problem of finding the optimum (in the least-squares sense) model field $\boldsymbol{B}^{\text {model }}$ which corresponds to the arbitrary field $\boldsymbol{B}$. Both vector fields are defined at $N$ points on the stellar surface and the weight function $\omega$ describes relative accuracy with which $\boldsymbol{B}$ is known in each surface element. The stellar surface is assumed to have the form of a sphere with radius $R_{\star}$.

After selecting an appropriate parametrization of the model field $\boldsymbol{B}^{\text {model }}$ the problem can be solved by minimizing the functional

$\mathfrak{R}=\sum_{i=1}^{N} \omega_{i} \sum_{j=1}^{3}\left(B_{i j}-B_{i j}^{\text {model }}\right)^{2}$,

where the index $j$ corresponds to three components of the vector field and the index $i$ runs over all surface elements.

In our study we represent the model field as a superposition of real spherical harmonics $S_{l m}(\theta, \phi)$ up to the $M$ th order:

$\boldsymbol{B}^{\text {model }}=-\nabla\left[\sum_{l=1}^{M} \sum_{m=-l}^{l} q_{l m} \frac{S_{l m}(\theta, \phi)}{r^{l+1}}\right]_{r=R_{\star}}$.

Real spherical harmonics are defined as (e.g. Varshalovich et al. 1988)

$S_{l m}(\theta, \phi) \equiv \begin{cases}C_{l m} P_{l}^{m}(\cos \theta) \cos (m \phi), & \text { for } m \geq 0 \\ C_{l m} P_{l}^{|m|}(\cos \theta) \sin (|m| \phi), & \text { for } m<0\end{cases}$

where normalization coefficients $C_{l m}$ are

$C_{l m} \equiv \begin{cases}\sqrt{\frac{2 l+1}{4 \pi}}, & \text { for } m=0 \\ \sqrt{\frac{2 l+1}{2 \pi} \frac{(l-|m|) !}{(l+|m|) !},} & \text { for } m \neq 0\end{cases}$

and $P_{l}^{m}(\cos \theta)$ is the associated Legendre polynomial. 
Real spherical harmonics $S_{l m}(\theta, \phi)$ form a complete set of orthogonal functions of spherical coordinates $\theta$ and $\phi$ and satisfy normalization relations

$\int_{0}^{2 \pi} \mathrm{d} \phi \int_{0}^{\pi} \mathrm{d} \theta \sin \theta S_{l^{\prime} m^{\prime}}(\theta, \phi) S_{l m}(\theta, \phi) \propto \delta_{l l^{\prime}} \delta_{m m^{\prime}}$.

With expansion (A.2) the functional (A.1) takes the form

$\mathfrak{R}=\sum_{i=1}^{N} \omega_{i} \sum_{j=1}^{3}\left(B_{i j}-\sum_{l=1}^{M} \sum_{m=l}^{l} q_{l m} \mathfrak{B}_{i j}^{l m}\right)^{2}$,

where the gradients of individual multipolar components are

$\mathfrak{B}_{i j}^{l m}=-\nabla_{j}\left[\frac{S_{l m}\left(\theta_{i}, \phi_{i}\right)}{r^{l+1}}\right]_{r=R_{\star}}$.

The expansion coefficients $q_{l m}$ can now be found from the system of $2 M(M+1)$ linear equations

$$
\frac{\partial \Re}{\partial q_{l m}}=0 \text {. }
$$

After rearrangement, the explicit form of the right-hand side of (A.8) is

$A_{l m}^{l^{\prime} m^{\prime}}=\sum_{i=1}^{N} \omega_{i} \sum_{j=1}^{3} \mathfrak{B}_{i j}^{l m} \mathfrak{B}_{i j}^{l^{\prime} m^{\prime}}$

and for the left-hand side we obtain

$C_{l m}=\sum_{i=1}^{N} \omega_{i} \sum_{j=1}^{3} B_{i j} \mathfrak{B}_{i j}^{l m}$.

Thus linear system (A.8) is reduced to the conventional form

$A q=C$

and the expansion coefficients $q_{l m}$ are determined by inverting the matrix $\boldsymbol{A}$,

$\boldsymbol{q}=\boldsymbol{A}^{-1} \boldsymbol{C}$.

In the special case when the weight function $\omega$ is constant over the whole stellar surface, normalization conditions which follow from (A.5) diagonalize matrix (A.9) and (A.12) reduces to

$q_{l m}=\frac{C_{l m}}{A_{l m}^{l m}}$.

Expansion (A.2) can be carried out in an arbitrary spherical coordinate system $(r, \theta, \phi)$ and allows quick, accurate and unique evaluation of the coefficients $q_{l m}$ for a given field distribution. If desired these coefficients can be translated into the equivalent set of polar strengths and orientations of the dipole, quadrupole and other multipolar field components. The expressions explaining a relation between the two parametrizations are given in Bagnulo et al. (1996).
Acknowledgements. We would like to thank Knut and Alice Wallenberg Foundation for contributing the state-of-the-art computing facilities at the Institute of Astronomy and Space Physics in Uppsala, and to Jean-François Donati and Martin Stift for very useful discussions about the concepts and numerical implementation of MDI.

\section{References}

Auer, L. H., Heasley, J. N., \& House, L. L. 1977, ApJ, 216, 531

Bagnulo, S., Landolfi, M., Mathys, G., \& Landi Degl'Innocenti, M. 2000, A\&A, 358, 929

Bagnulo, S., Landi Degl'Innocenti, M., \& Landi Degl'Innocenti, E. 1996, A\&A, 308, 115

Berdyugina, S. V. 1998, A\&A, 338, 97

Bevington, P. R., \& Robinson, D. K. 1992, Data Reduction and Error Analysis for the Physical Sciences (McGraw-Hill, New-York)

Brown, S. F., Donati, J.-F., Rees, D. E., \& Semel, M. 1991, A\&A, 250, 463

Deutsch, A. 1958, Electromagnetic Phenomena in Cosmical Physics, ed. B. Lehnert, Stockholm 1956 (Cambridge Univ. Press), IAU Symp., 6, 209

Donati, J.-F. 2001, in the Proc. of First International Workshop on Astro-Tomography, ed. H. Boffin, D. Steeghs, \& J. Cuypers (Springer, Berlin), in press

Donati, J.-F., Achilleos, N., Matthews, J. M., \& Wesemael, F. 1994, A\&A, 285, 285

Donati, J.-F., \& Brown, S. F. 1997, A\&A, 326, 1135

Donati, J.-F. 1999, MNRAS, 302, 457

Donati, J.-F., Cameron, A. C., Hussain, G. A. J., \& Semel, M. 1999, MNRAS, 302, 437

Fensl, M. 1995, A\&AS, 112, 191

Goncharsky, A. V., Stepanov, V. V., Khokhlova, V. L., \& Yagola, A. G. 1982, Astron. Zh., 59, 1146

Humlíček, J. 1982, JQSRT, 27, 437

Hussain, G. A. J., Donati, J.-F., Cameron, A. C., \& Barnes, J. 2000, MNRAS, 318, 961

Kemp, J. C. 1970, ApJ, 162, 169

Khokhlova, V. L., Vasilchenko, D. V., Stepanov, V. V., \& Tsymbal, V. V. 1997, Astron. Lett., 23, 465

Kupka, F., Piskunov, N., Ryabchikova, T. A., Stempels, H. C., \& Weiss, W. W. 1999, A\&AS, 138, 119

Kurucz, R. 1993, CD-ROM No. 13, Smithsonian Astrophys. Obs.

Landi Degl'Innocenti, E. 1976, A\&AS, 25, 379

Landstreet, J. D. 1988, ApJ, 326, 967

Landstreet, J. D., \& Mathys, G. 2000, A\&A, 359, 213

Piskunov, N. E. 1985, Astron. Lett., 10, 449

Piskunov, N. 1992, in the Proc. of the International meeting Stellar Magnetism, Russian Special Astrophys. Observatory, Nizhnij Arkhiz, 1991, ed. Yu. V. Glagolevskij, \& I. I. Romanuk (Nauka: St.Petersburg), 92

Piskunov, N. 1999, in the Proc. of the 2nd Workshop on Solar Polarization, ed. J. O. Stenflo, \& K. N. Nagendra 1998, Bangalore, India (Kluwer: Dordrecht), 515

Piskunov, N., Kupka, F., Ryabchikova, T. A., Weiss, W. W., \& Jeffery, C. S. 1995, A\&AS, 112, 525

Piskunov, N., \& Rice, J. B. 1993, PASP, 105, 1415

Piskunov, N. E., Tuominen, I., \& Vilhu, O. 1990, A\&A, 230, 363 
Rees, D. E., \& Murphy, G. A. 1987, Numerical Radiative Transfer, ed. W. Kalkofen (Cambridge University Press), 241

Rees, D. E., Murphy, G. A., \& Durrant, C. J. 1989, ApJ, 339, 1093

Rice, J. B., Wehlau, W. H., \& Khokhlova, V. L. 1989, A\&A, 208, 179

Ruiz Cobo, B., Bellot Rubio, L. R., \& Collados, M. 1999, in the Proc. of the 2nd Workshop on Solar Polarization, ed. J. O. Stenflo, \& K. N. Nagendra 1998, Bangalore, India (Kluwer: Dordrecht), 231

Semel, M., Donati, J.-F., \& Rees, D. E. 1993, A\&A, 278, 231

Skilling, J., \& Bryan, R. J. 1984, MNRAS, 211, 111

Socas-Navarro, H., Trujillo Bueno, J., \& Ruiz Cobo, B. 2000, ApJ, 530, 977
Stenflo, J. O., Gandorfer, A., Wenzler, T., \& Keller, C. U. 2001, A\&A, 367, 1033

Tikhonov, A. N., \& Arsenin, V. Y. 1977, Solution of ill-posed problems (Wiley: New York)

Varshalovich, D. A., Moskalev, A. N., \& Khersonskii, V. K. 1988, Quantum theory of angular momentum (World Scientific, Singapore)

Vasilchenko, D. V., Stepanov, V. V., \& Khokhlova, V. L. 1996, Pis'ma AZh, 22, 924

Vogt, S. S., Penrod, G. D., \& Hatzes, A. P. 1987, ApJ, 321, 496

Wade, G. A., Bagnulo, S., Kochukhov, O., et al. 2001, A\&A, 374,265 